\title{
Increasing the accuracy and temporal resolution of two-filter radon-222 measurements by correcting for the instrument response
}

\author{
Alan D. Griffiths, Scott D. Chambers, Alastair G. Williams, and Sylvester Werczynski \\ Australian Nuclear Science and Technology Organisation, Locked Bag 2001, Kirrawee DC NSW 2232, Australia \\ Correspondence to: Alan D. Griffiths (alan.griffiths@ansto.gov.au)
}

Received: 7 December 2015 - Published in Atmos. Meas. Tech. Discuss.: 19 January 2016

Revised: 24 May 2016 - Accepted: 26 May 2016 - Published: 28 June 2016

\begin{abstract}
Dual-flow-loop two-filter radon detectors have a slow time response, which can affect the interpretation of their output when making continuous observations of nearsurface atmospheric radon concentrations. While concentrations are routinely reported hourly, a calibrated model of detector performance shows that $\sim 40 \%$ of the signal arrives more than an hour after a radon pulse is delivered. After investigating several possible ways to correct for the detector's slow time response, we show that a Bayesian approach using a Markov chain Monte Carlo sampler is an effective method. After deconvolution, the detector's output is redistributed into the appropriate counting interval and a $10 \mathrm{~min}$ temporal resolution can be achieved under test conditions when the radon concentration is controlled. In the case of existing archived observations, collected under less ideal conditions, the data can be retrospectively reprocessed at $30 \mathrm{~min}$ resolution. In one case study, we demonstrate that a deconvolved radon time series was consistent with the following: measurements from a fast-response carbon dioxide monitor; grab samples from an aircraft; and a simple mixing height model. In another case study, during a period of stable nights and days with well-developed convective boundary layers, a bias of $18 \%$ in the mean daily minimum radon concentration was eliminated by correcting for the instrument response.
\end{abstract}

\section{Introduction}

Radon-222 (radon) is a radioactive noble gas emitted primarily from the ice-free land surface, and widely used as a natural passive tracer in the atmosphere. It is almost ideal as a passive tracer; as well as being chemically inert it has a relatively well-characterised terrestrial source, and a short 3.8-day half-life. Radon measurements have been used to identify baseline air masses at atmospheric monitoring stations (Brunke et al., 2004; Chambers et al., 2014, 2015a; Xia et al., 2013), for model validation (Considine et al., 2005; Jacob and Prather, 1990; Zhang et al., 2008), and for making land surface flux estimates (Biraud et al., 2000; Slemr et al., 2013; van der Laan et al., 2014). Radon has also been used as a tracer in recent studies of vertical mixing (Chambers et al., 2011; Griffiths et al., 2013; Williams et al., 2011, 2013; Vargas et al., 2015) and in many earlier studies, some of which are reviewed by Zahorowski et al. (2004).

One of the challenges of using radon as a tracer is that emissions are small ( $\sim 1$ atom $\mathrm{cm}^{-2} \mathrm{~s}^{-1}$; Zhang et al., 2011) and ambient concentrations are low. Low-level atmospheric measurements are therefore a niche served largely by instruments built in-house (Frank et al., 2012; Levin et al., 2002; Schmithüsen et al., 2016; Wada et al., 2010, 2012; Whittlestone and Zahorowski, 1998; Grossi et al., 2012). The dual-flow-loop two-filter radon detector (Whittlestone and Zahorowski, 1998; Chambers et al., 2011) is the focus of this paper. It is based on the two-filter method for radon measurements (Thomas and Leclare, 1970) and is called the "twofilter detector" for short.

An advantage of the two-filter detector is that it provides a direct measure of radon concentration, rather than inferring it from the ambient concentration of radon progeny. Although direct measurements are common for measuring higher radon concentrations, for instance when measuring radon emissions from soil (Grossi et al., 2011; Zahorowski and Whittlestone, 1996), progeny measurements are often used for low-concentration measurements because it is possible to increase the measurement sensitivity by accumulating progeny by filtration or electrostatic precipitation. When 
progeny are trapped directly from ambient air; e.g. using the one-filter method, the derived radon concentration is more sensitive to environmental parameters than two-filter detectors (Xia et al., 2010). This is because the two-filter detector is unaffected by the degree of ambient disequilibrium between radon and its progeny (equilibrium, in a radioactive decay chain, is when the activity concentration of a parent equals the activity concentration of its progeny). Even though the two-filter detector measures the alpha activity of radon progeny internally, these progeny are generated under controlled conditions inside a delay chamber, so that steady-state counts are proportional to the ambient radon concentration. The cost of being sensitive to radon concentration alone is that the detector is large, to accommodate the delay chamber, and has the opportunity to see fewer counts than progeny detectors because of practical limits on the size of the delay chamber.

Radon detectors are important because of an increasing need for reliable measurements. Radon emissions, which must be known for model validation studies, are increasingly well-characterised (e.g. Karstens et al., 2015) and radon applications are becoming more ambitious (Chambers et al., 2015a; Vogel et al., 2013). In contrast, prior studies made simple assumptions about radon emissions (Conen and Robertson, 2002) and focussed on clear trends, such as identifying the contrast between boundary-layer air and the radon-depleted free troposphere (Gäggeler et al., 1995; Guedalia et al., 1972).

Although sensitive and precise enough for the studies mentioned above, a major limitation of the two-filter detector is its slow temporal response, with a one-half rise time of about $45 \mathrm{~min}$. The slow detector response is a problem common to many high-sensitivity techniques. It puts a limit on the detector's usefulness during non-stationary conditions, most strikingly at inland sites when radon concentrations drop rapidly following the morning onset of vertical mixing. This makes it difficult to compare radon measurements with flask samples, or with measurements of other quantities from fast-response instruments.

In order to tackle this problem, we have implemented a deconvolution method to correct observations for the instrument response. Deconvolution is a problem which arises frequently in atmospheric measurements because of the finite spatial or temporal response of sensors. For example, deconvolution methods have been applied to eddy correlation (Moore, 1986; Massman, 2000), cavity ring-down spectroscopy (Winderlich et al., 2010), temperature (McCarthy, 1973) and radiation measurements (Ehrlich and Wendisch, 2015). In our favoured implementation, the measurement uncertainty is estimated, along with the reconstructed time series, and care is taken to avoid the amplification of measurement noise.

The purpose of this paper is to describe the deconvolution method and the effects of deconvolution on the interpretation of several case studies. In Sect. 2 a mathemati-

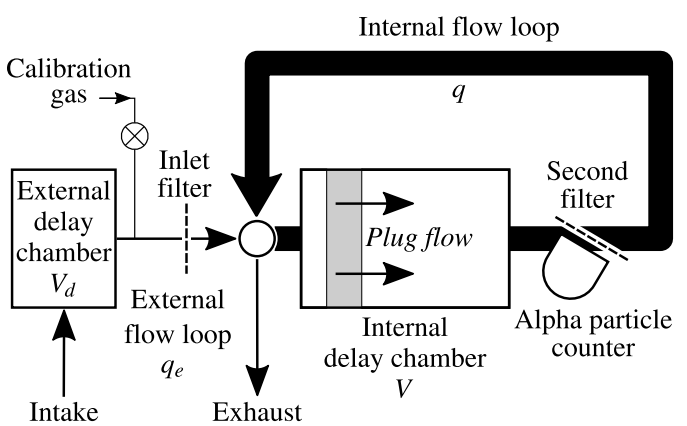

Figure 1. Process diagram of the dual-flow-loop two-filter radon detector. Wider arrows indicate higher flow rates. For the detector used in these tests, the inlet filter was a high efficiency particulate filter (Vokes Air Hepatex JK), and the second filter was a twill weave stainless steel mesh made from $20 \mu$ diameter wire with $20 \mu$ openings (635 mesh, TWP Inc.). The alpha particle counter consisted of an assembly holding the filter, silver activated zinc sulfide scintillation paper, and photomultiplier (9330B, ET Enterprises).

cal model of the detector is developed and used to explain the detector response to a radon pulse. In Sect. 3, deconvolution methods of increasing complexity are described. Three methods are discussed: Richardson-Lucy deconvolution; Richardson-Lucy deconvolution with total variation regularisation; and a Bayesian Monte Carlo technique. The favoured method, Bayesian Monte Carlo deconvolution, uses the simpler Richardson-Lucy method in its implementation. In Sect. 4 the deconvolution methods are tested under controlled conditions. In this test, an hour-long pulse of radon is delivered to the detector and reconstructed at 10-min resolution. Then, in Sect. 6, the deconvolution method is applied to data acquired in a previous field campaign. As well as radon observations, the interpretation draws on a range of micrometeorological data from surface and aircraft sensors. In Sect. 7 the need for deconvolution is discussed, along with suggestions for detector operation which improve the chances of successfully correcting the detector output. Finally, conclusions are drawn in Sect. 8.

\section{Radon detector model}

This section describes a simple model of the dual-flow-loop two-filter radon detector (Fig. 1). The model is based on the one described by Whittlestone and Zahorowski (1998), but with additions which were needed for an unbiased fit to observations necessary for deconvolution. The model is described in full alongside a description of the detector itself. At the end of the section, model output is compared with experiments. 


\subsection{Operating principles}

The two-filter detector operates by drawing ambient air through a particulate filter, to remove ambient radon progeny, and then counting radon progeny on a second filter which is downstream of a delay chamber. The only source of progeny, on the second filter, is the radioactive decay of radon in the delay chamber. The count rate, as a result, is proportional to the ambient radon concentration.

The delay chamber volume is proportional to the desired sensitivity. Detectors have been built with delay chambers of nominal volumes $100,700,1500$, and $5000 \mathrm{~L}$, the smallest intended for inland use (Martin et al., 2004) and the largest for remote stations which monitor non-terrestrial air (Zahorowski et al., 2013, e.g.). By optimising other aspects of the design (Whittlestone et al., 1994), the measured net efficiency has proven to scale roughly linearly with volume, $\epsilon_{\text {net }} \approx 0.2 V$ where $\epsilon_{\text {net }}$ is the number of counts per second at an ambient concentration of $1 \mathrm{~Bq} \mathrm{~m}^{-3}$ and $V$ is the internal volume in $\mathrm{m}^{3}$. We focus on the $700 \mathrm{~L}$ detector in this paper but also show an example from a $1500 \mathrm{~L}$ detector.

Because the detector works by gross alpha counting, the signal is also sensitive to thoron (radon-220, with a halflife of $56 \mathrm{~s}$ ). Like radon, thoron is a gas that is emitted from the ground. Its decay products include an alpha-emitting daughter (lead-212, with a half-life of $10.6 \mathrm{~h}$ ) that causes an elevated background if allowed to reach the second filter (Williams and Chambers, 2016). For installations where thoron contamination is possible, the thoron signal is attenuated by passing sample air through an external delay chamber before entering the detector, aiming for a delay of at least $5 \mathrm{~min}$.

Calibration is usually performed monthly by injecting radon from a calibrated source (typically with a $5 \%$ absolute uncertainty), and the background count rate measured quarterly. For more about a typical installation, see Chambers et al. (2011).

\subsection{Model formulation}

Whittlestone and Zahorowski (1998) present a model which describes the detector well enough to estimate its most important operational characteristics, such as the net efficiency and response time. Extra terms need to be introduced in order to reproduce day-to-day variations in the detector response. Day-to-day variations are small, but important for estimating the deconvolution uncertainty, so these extra terms are included in the present application.

Even with these extra terms, the model includes several simplifying assumptions meaning that it is far from a comprehensive treatment of processes within the detector. This suits our needs, as the purpose of the model is utilitarian; it needs to be able to reproduce the time-response of the detector, including day-to-day changes, preferably with few adjustable parameters. In the next section, validation data are

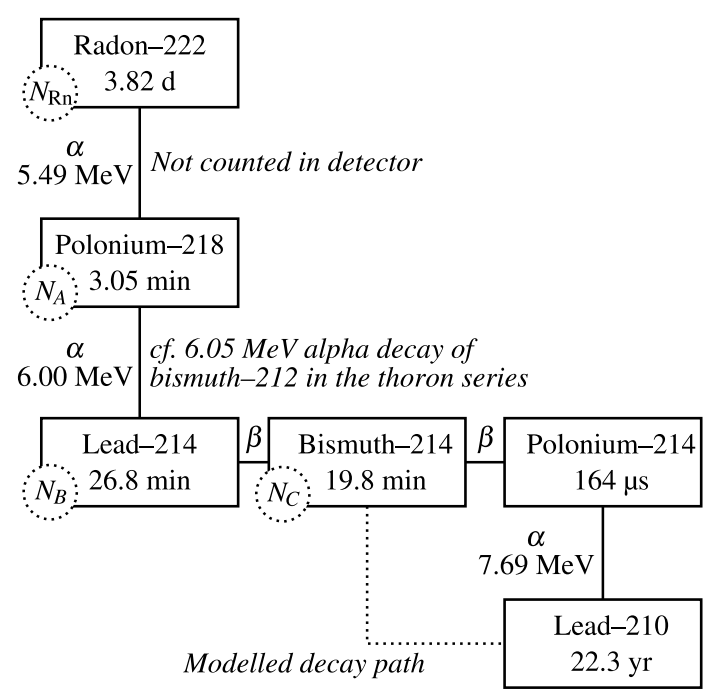

Figure 2. The decay series of radon and its immediate progeny, an extract from the uranium-238 series. The figure shows the energy of alpha decays, and nomenclature used to identify species abundances: $N_{\mathrm{Rn}}, N_{A}, N_{B}$, and $N_{C}$.

presented to show that the model meets these requirements. Here, the model is described in full.

Following the notation of Whittlestone and Zahorowski (1998), radioelement concentrations in air are given in number densities (atoms $\mathrm{m}^{-3}$ ), using the symbols shown in Fig. 2: $N_{\mathrm{Rn}}$ for radon-222, $N_{A}$ for polonium-218, $N_{B}$ for lead-214, and $N_{C}$ for bismuth-214. Bismuth-214 decays are treated as immediate alpha decays to stable lead210 , because of the short half-life of the intermediate species, polonium-214. The radioactive decay constant, $\lambda=$ $\log (2) / \tau$, where $\tau$ is the half life, is written with the same subscript as the element's number density.

Flow rates in the model are volumetric with units of $\mathrm{m}^{3} \mathrm{~s}^{-1}$ at the temperature and pressure of the delay chamber. Number densities are also referenced to the delay chamber conditions. Temperature and pressure are logged in the delay chamber to allow measured radon concentrations to be converted to STP conditions (standard temperature and pressure, $20^{\circ} \mathrm{C}, 1000 \mathrm{hPa}$, written $\mathrm{Bq} \mathrm{m}^{-3} \mathrm{STP}$ ) or to ambient conditions, if ambient temperature and pressure are logged with a separate weather station. Other routinely logged parameters include the internal and external flow rate and the relative humidity within the delay chamber.

As shown in Fig. 1, ambient air is drawn first into an external delay chamber, at the external flow rate of $q_{\mathrm{e}}$, to remove thoron. Ambient air has a radon concentration $N_{\mathrm{Rn}_{\mathrm{e}}}$, where the units are activity per unit volume at the delay chamber temperature and pressure, and the external delay chamber has a radon concentration $N_{\mathrm{Rn}_{\mathrm{d}}}$ and volume $V_{\mathrm{d}}$. In the model, the air in the delay chamber is always completely mixed and the temperature changes from the external air temperature, $T_{\mathrm{e}}$, to 
the internal detector temperature, $T_{\mathrm{i}}$, instantaneously at the detector inlet.

In the delay chamber, the balance between radon inflow, outflow, and decay is

$\frac{\mathrm{d} N_{\mathrm{Rn}_{\mathrm{d}}}}{\mathrm{d} t}=\frac{q_{\mathrm{e}}}{V_{\mathrm{d}}}\left(N_{\mathrm{Rn}_{\mathrm{e}}}-N_{\mathrm{Rn}_{\mathrm{d}}}\right)-N_{\mathrm{Rn}_{\mathrm{d}}} \lambda_{\mathrm{Rn}}$.

Air exiting the external delay chamber passes through the first filter; which removes radon progeny, thoron progeny, and aerosols; then enters the main flow loop. The radon concentration, $N_{\mathrm{Rn}}$, is assumed to be uniform throughout the internal delay chamber because of the rapid circulation of air $\left(q \gg q_{\mathrm{e}}, q / V \approx 1 \mathrm{~min}\right)$. In the internal delay chamber,

$$
\begin{aligned}
\frac{\mathrm{d} N_{\mathrm{Rn}}}{\mathrm{d} t} & =\frac{q_{\mathrm{e}}}{V}\left(N_{\mathrm{Rn}_{\mathrm{d}}}+N_{\mathrm{Rn}_{\mathrm{cal}}}-N_{\mathrm{Rn}}\right) \\
& -N_{\mathrm{Rn}} \lambda_{\mathrm{Rn}}-N_{\mathrm{Rn}} \frac{1}{T} \frac{\mathrm{d} T}{\mathrm{~d} t} .
\end{aligned}
$$

Equation (2) differs from the Whittlestone and Zahorowski (1998) model in two ways. First, the last term in Eq. (2) takes into account the difference between flow rates in and out of the internal delay chamber as a result of a temperatureinduced trend in air density. It causes, at most, a $\sim 2 \%$ change in detector output when the detector temperature is changing rapidly. For detectors installed indoors, it could be neglected. Second, Eq. (2) has been modified to simulate detector calibration. During normal use, the valve between the delay chamber and the calibration source (Fig. 1) is closed and $N_{\mathrm{Rn}_{\text {cal }}}=0$. During calibration, $N_{\mathrm{Rn}_{\text {cal }}}=A_{\mathrm{s}} / q_{\mathrm{e}}$, where $A_{\mathrm{S}}$ is the calibration source activity. The calibration source is chosen so that $N_{\mathrm{Rn}_{\text {cal }}} \gg N_{\mathrm{Rn}}$.

Returning to the description of usual operation: plug flow is assumed to exist within the internal delay chamber; the internal flow rate is chosen so that the mean transit time, $\tau_{\mathrm{t}}=V / q$, through the delay chamber is about 1 minute; and inside the internal delay chamber, radon atoms decay according to the series shown in Fig. 2. Unattached radon daughters deposit on chamber walls, or plate out, with a deposition velocity, $v_{\mathrm{d}}$, of about $0.15-0.5 \mathrm{~mm} \mathrm{~s}^{-1}$, depending on the circulation pattern (McLaughlin and O'Byrne, 1984; Nazaroff et al., 1992). Deposition is parametrised here as an exponential loss process, with a single time constant of $\lambda_{p}$ for all species. The time constant $\lambda_{\mathrm{p}}$ can be linked to the more usual parameterisation of deposition, based on the deposition velocity $v_{\mathrm{d}}$, by equating the expressions for the volumeintegrated deposition, $V \lambda_{\mathrm{p}} N_{A}$, with the area-integrated deposition $A v_{\mathrm{d}} N_{A}$, where $A$ is the internal surface-area of the delay chamber. Hence, $\lambda_{\mathrm{p}}=A v_{\mathrm{d}} / V$.

Because plug flow is assumed, it is possible to consider an air parcel which travels, without mixing, through the delay chamber. It enters the delay chamber at time $t=0$, free of radon progeny. Then, as it transits the delay chamber, the progeny concentration evolves according to

$$
\begin{aligned}
\frac{\mathrm{d} N_{A}}{\mathrm{~d} t} & =N_{\mathrm{Rn}} \lambda_{\mathrm{Rn}}-N_{A}\left(\lambda_{A}+\lambda_{\mathrm{p}}\right) \\
\frac{\mathrm{d} N_{B}}{\mathrm{~d} t} & =N_{A} \lambda_{A}-N_{B}\left(\lambda_{B}+\lambda_{\mathrm{p}}\right) \\
\frac{\mathrm{d} N_{C}}{\mathrm{~d} t} & =N_{B} \lambda_{B}-N_{C}\left(\lambda_{C}+\lambda_{\mathrm{p}}\right) .
\end{aligned}
$$

In air arriving at the second filter, the concentration of radon progeny is given by the solution to Eqs. (3)-(5) at a time equal to the transit time, $t=\tau_{\mathrm{t}}$, with $N_{A}=N_{B}=N_{C}=$ 0 at $t=0$. The solution can be found numerically, or written analytically. Because of its length, it is not reproduced here but can be found in the Supplement.

Due to the presence of the first filter, and the short transit time, polonium-218 (species A) is the dominant species trapped on the second filter, which is actually a fine wire screen. Because aerosols are excluded from the tank, radon progeny exists as unattached clusters which are efficiently trapped on the screen due to their large diffusivity. The efficiency of the screen; $\epsilon_{\mathrm{s}}$, discussed in Sect. 2.3 ; is $\gtrsim 95 \%$. Air re-entering the delay chamber needs to pass through a length of pipe, a blower, and a denim screen, all of which trap progeny and ensure that air re-entering the delay chamber is, to a good approximation, progeny-free.

The number of progeny, $F_{A}, F_{B}$, and $F_{C}$, resident on the screen is determined by a balance between the arrival of new progeny, trapped at a rate $q \epsilon_{\mathrm{s}} N$, in situ production from the decay of parent isotopes, and loss processes. Loss processes are dominated by radioactive decay, but an additional loss process, not included by Whittlestone and Zahorowski (1998), is recoil loss (Jonassen and McLaughlin, 1976). During alpha decay, the nucleus of the newly formed progeny recoils away from the alpha particle to conserve momentum. Although momentum is conserved for beta and gamma emission too, only in the case of alpha decay is enough kinetic energy transferred to the nucleus for it to be lost from the screen. In the detector, recoil is significant only for the decay of polonium-218 (species A), whose probability of being lost from the screen is written $p_{\mathrm{r}}$. These considerations lead to

$$
\begin{aligned}
\frac{\mathrm{d} F_{A}}{\mathrm{~d} t} & =q \epsilon_{\mathrm{s}} N_{A}-F_{A} \lambda_{A} \\
\frac{\mathrm{d} F_{B}}{\mathrm{~d} t} & =q \epsilon_{\mathrm{s}} N_{B}+\left(1-p_{r}\right) F_{A} \lambda_{A}-F_{B} \lambda_{B} \\
\frac{\mathrm{d} F_{C}}{\mathrm{~d} t} & =q \epsilon_{\mathrm{s}} N_{C}+F_{B} \lambda_{B}-F_{C} \lambda_{C} .
\end{aligned}
$$

A silver activated zinc sulphide scintillator and photomultiplier tube assembly count alpha particles with an efficiency $\epsilon_{\mathrm{d}}$, which is assumed to take a single value for both alpha particles. This is an approximation, because alpha particles from the two species have different energies. Polonium-218 (species A) decays with alpha particles which are $22 \%$ less energetic, have a shorter range in air, and are slightly less 
likely to reach the scintillator. In the simplified decay chain, this gives a detector activity (alpha particles counted per second) of

$A_{\mathrm{d}}=\epsilon_{\mathrm{d}}\left(F_{A} \lambda_{A}+F_{C} \lambda_{C}\right)$,

which is integrated over a counting interval, $\delta t$. Typically, $\delta t=30 \mathrm{~min}$ for comparison with observations. Although there are theoretical estimates for the counts expected from each side of a screen with simple geometry (Solomon and Ren, 1992), $\epsilon_{\mathrm{d}}$ is effectively a free parameter in this model because the geometry of the screen and photomultiplier assembly is complicated, the design having been improved over several iterations (Whittlestone and Zahorowski, 1998; Williams and Chambers, 2016).

An important characteristic of the detector, the net steadystate efficiency, $\epsilon_{\text {net }}$, is found by setting time derivatives to zero, combining Eqs. (6)-(9), and computing the count rate for an inflow radon concentration of $1 \mathrm{~Bq} \mathrm{~m}^{-3}$. Although $\epsilon_{\text {net }}$ depends on several model parameters, it is controlled in practice by the delay volume, $V$, provided that the flow rate $q$ is adjusted so that the screen efficiency is high and the plateout loss is small (Sect. 2.1).

We implemented this model using the Boost odeint ordinary differential equation solver (Ahnert and Mulansky, 2011). Ambient radon concentrations were represented as a piecewise linear or piecewise constant time series, depending on the case being simulated.

\subsection{Environmental influences on model parameters}

Some of the model parameters are potentially affected by changes to the environment within the delay chamber. In the delay chamber, radon atoms decay by alpha emission into polonium-218. Initially, $88 \%$ of the polonium atoms carry a positive charge (Porstendörfer, 2001) but lose their charge so that the steady-state fraction of charged clusters is roughly $50 \%$ (Dankelmann et al., 2001). Both charged and neutral polonium atoms form unattached clusters with an aerodynamic size of about $1 \mathrm{~nm}$ (Zhang et al., 2014), but charged atoms form larger clusters with a lower diffusivity. Because the neutralisation rate depends on relative humidity, at least in the presence of reactive trace gases (Frey et al., 1981), so the net diffusivity of polonium-218, and other radon daughters, can change with relative humidity. This is a potential cause of changes in both the screen efficiency, $\epsilon_{\mathrm{s}}$, and the plateout time constant, $\lambda_{\mathrm{p}}$, both of which increase with diffusivity. The effect of humidity is important only in the range between about 0 and $30 \%$ (Su et al., 1988), asymptotically approaching a constant value at higher relative humidities. This range of sensitivity to humidity compares well with the results of Thomas and Leclare (1970), who saw an effect on their two-filter apparatus in the range 0-20\%.

Based on particle diffusivity, the efficiency of woven steel screens can be computed from theory (Cheng et al., 1980; Cheng and Yeh, 1980). The Cheng et al. theory has been validated experimentally for particles above about $2 \mathrm{~nm}$ (Scheibel and Porstendörfer, 1984; Ichitsubo et al., 1996; Alonso et al., 1997; Heim et al., 2005; Shin et al., 2008; Thomas et al., 2013) and, although it begins to under-predict the screen collection efficiency for smaller particles (Heim et al., 2010), is appropriate for radon progeny. For the flow rate typical in the $700 \mathrm{~L}$ detector, $q=12.2 \mathrm{~L} \mathrm{~s}^{-1}$, a filter area of $262 \mathrm{~cm}^{2}$, and taking the diffusivity of neutral clusters to be $0.08 \mathrm{~cm}^{2} \mathrm{~s}^{-1}$ and positive clusters to be $0.03 \mathrm{~cm}^{2} \mathrm{~s}^{-1}$ (Frey et al., 1981), $\epsilon_{\mathrm{s}}=0.98$ for neutral clusters and 0.87 for positive clusters. Bearing in mind an upper limit of $88 \%$ positive clusters, the net value of $\epsilon_{\mathrm{S}}$ should lie in the range 0.89-0.98, assuming uniform flow across the screen.

In isolation, a change in the screen efficiency, though it affects counting efficiency, will not lead to a change in the shape of the detector response. To match the observed changes in detector response, there needs to be a process which allows atoms to be lost from the screen at a varying rate. A parameter which allows this is a changing recoil fraction. The upper limit of this effect is for a flat surface in a vacuum, where there is a $50 \%$ chance of the recoil velocity being directed away from the surface and an atom being lost (Jonassen and McLaughlin, 1976). For a screen in an airflow, the recoil losses measured by Knutson and George (1994) were consistent with there being a $50 \%$ chance of recoil loss followed by re-attachment to the screen at the same rate as the screen efficiency. Although based on only four samples, the Knutson and George (1994) results suggest that the recoil probability can be written

$p_{\mathrm{r}}=\frac{1}{2}\left(1-\epsilon_{\mathrm{s}}\right)$.

Following this chain of reasoning, we can expect the radon detector's response to have more weight at long lag times when $p_{\mathrm{r}}$ is small, which will be more likely on days when the delay chamber humidity is above $\sim 20 \%$. Based on the expected range for $\epsilon_{\mathrm{s}}$ and Eq. (10), $p_{\mathrm{r}}$, should lie in the range $0-0.055$.

\subsection{Model validation}

The main purpose of the detector model presented in Sect. 2.2 is to simulate the detector response, necessary for deconvolution as described later in Sect. 3. Some of the model parameters have to be fitted to experimental data, ideally to measurements of the detector response to a short pulse of radon. Such an experiment is presented here in order to demonstrate the validity of the detector model.

The modelled detector response consists of two overlapping peaks (Fig. 3a); counts occurring during the first hour are dominated by the decay of polonium-218, later counts are mainly from the decay of polonium-214. The relative timing of the two peaks is mainly controlled by the radioactive lifetime of the intermediate isotopes. In contrast, the 

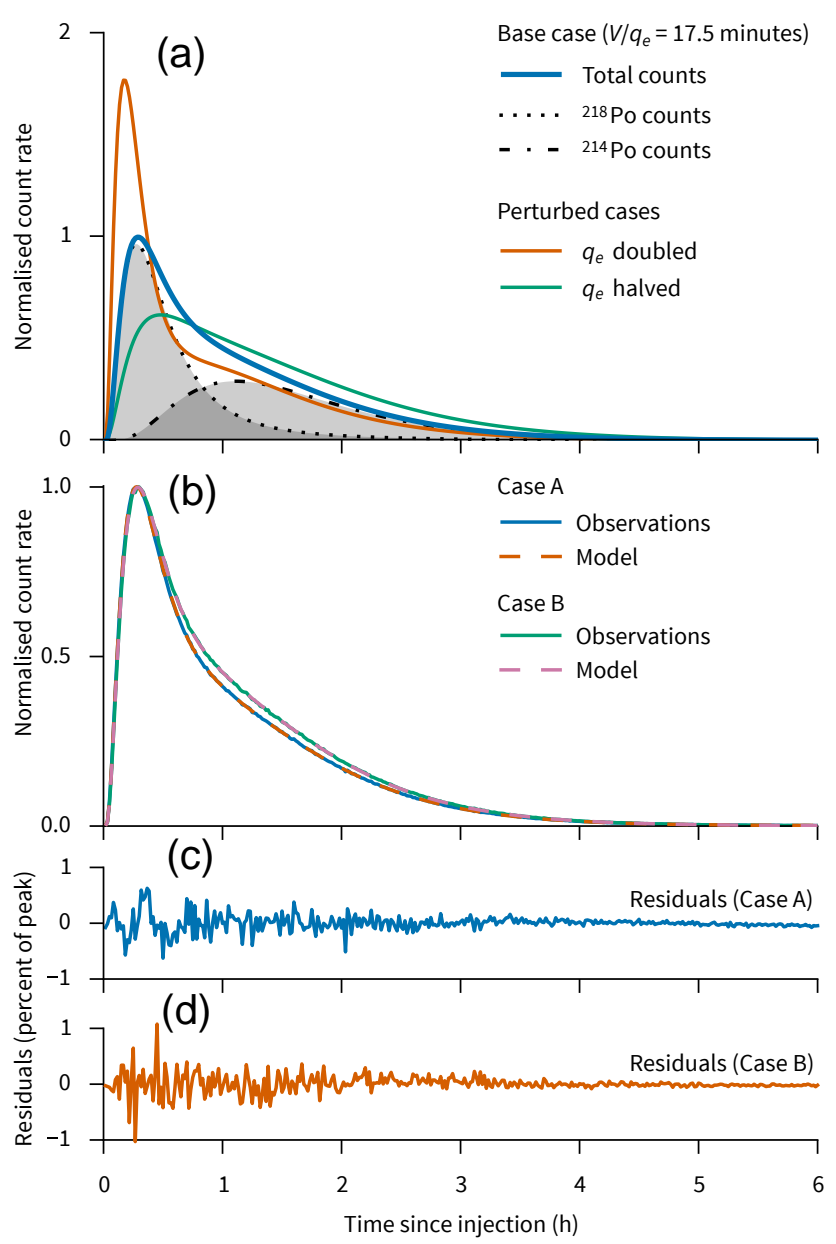

Figure 3. Modelled and observed detector response to a radon source injected for $1 \mathrm{~min}$ at time zero. Panel (a) shows the simulated response of the $700 \mathrm{~L}$ radon detector during normal operation (base case) including a break down between contributions from polonium-218 and polonium-214 counts. Also shown is the effect of changing the external flow rate, $q_{\mathrm{e}}$. Panel (b) shows the observed and modelled detector response, comparing 2 days which have differing detector responses, interpreted as a change in the fraction of polonium-218 progeny lost from the screen after alpha decay. Panels (c) and (d) show the residuals (observed minus modelled counts) after fitting the modelled response to observations. The pulse concentration is not precisely known, and not necessarily the same each day, so the detector response is normalised (by total counts in $\mathbf{a}$ and by peak counts in $\mathbf{b}$ ). The normalisation factor, in $\mathbf{b}$, is approximately $1 /\left(1500\right.$ count $\left.^{-1}\right)$. Over eight injections, the number of counts $0-5 \mathrm{~h}$ after injection has a coefficient of variation of $3 \%$.

shape of the first peak is strongly controlled by the inlet flow rate, $q_{\mathrm{e}}$, where a higher flow rate leads to a sharper peak.

So as to reproduce the large temperature variations seen in the field, for the validation experiments the detector was installed outdoors with full exposure to the midday sun. Ambient air was sampled continuously at about $2 \mathrm{~m}$ a.g.l. and, for $1 \mathrm{~min}$, radon was injected into the intake line upstream of the thoron delay chamber. According to specifications (Pylon Inc.) the source emits radon at $30.8 \mathrm{~Bq} \mathrm{~min}^{-1} \pm 5 \%$ when flushed continuously. By leaving the source sealed in between pulses (one per day at 13:00 LT; valves were controlled by a timer), radon accumulated inside the source, leading to a pulse which was much larger than the ambient radon concentration, although not precisely known. Counts were recorded every minute and the count rate peaked at $\sim 1500 \mathrm{~s}^{-1}$, compared with ambient counts of $\sim 1 \mathrm{~s}^{-1}$. For this $700 \mathrm{~L}$ detector, the count rate peaks at $18 \mathrm{~min}$ after injection and $50 \%$ of the counts occur before $49 \mathrm{~min} ; 42 \%$ of the counts are recorded after the first hour. The full-width at half-maximum is $47 \mathrm{~min}$ and the peak is asymmetric; the count rate is $50 \%$ of its maximum $11 \mathrm{~min}$ before the peak and $36 \mathrm{~min}$ after the peak.

The theoretical model, when fit to the observed data, has residuals which show little systematic structure (two cases are shown in Fig. 3). The Bayesian deconvolution approach was used to fit the model to observations. The method, described in Sect. 3.3, was modified for this purpose by specifying a known radon concentration along with uninformative priors for the model parameters without direct observations. Non-Bayesian optimisation, using Powell's method (Powell, 1964; Press et al., 2007), gave similar results.

The size of the model error is better than the typical counting error under ambient conditions, which indicates that the model is good enough to use for deconvolution. The residuals of Case A show what might be systematic structure around the peak, perhaps because of air flow variations, but the magnitude is small and model error is neglected in the following sections.

Day to day variations in the detector response can be matched by changing model parameters. The two cases in Fig. 3, marked "a" and "b", were chosen because they lie at two extremes of the observed ratio of polonium-214 to polonium-218 counts. To achieve a good fit on both of these days, the recoil parameter had to be allowed to vary.

Considering all nine of the pulse tests, the ratio of polonium-214 to polonium-218 counts tends to be higher on days when the relative humidity inside the detector is low (Fig. 4), which is consistent with a value of $p_{\mathrm{r}}$ at a humidity below $20 \%$.

To fit these observations, the recoil parameter, $p_{\mathrm{r}}$, must be varied within the range from 0 to 0.1 . This range is not wildly unrealistic, yet is larger than the range expected from theory $\left(0-0.055\right.$, Sect. 2.3). The reason for this is perhaps that $p_{\mathrm{r}}$ is acting as a proxy for an unknown process, or because of the known approximations in the model. Some of the approximations are that $\epsilon_{\mathrm{d}}$ and $\lambda_{\mathrm{p}}$ take a single value for all radon daughters, or the convenient assumptions of plug flow in the internal delay chamber and complete mixing in the external delay chamber. Dealing with this by adding complexity to the model is undesirable because there are already more parameters than can be constrained by observations. We therefore 


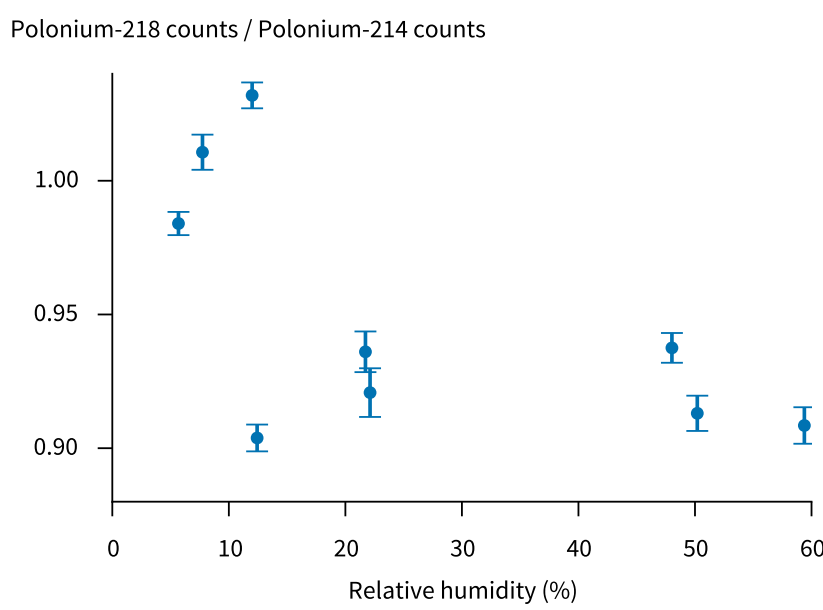

Figure 4. Ratio of polonium-218 to -214 counts (related to the recoil probability, $p_{\mathrm{r}}$ ) during pulse injection tests as a function of the average relative humidity within the detector for the first hour since pulse injection. The Bayesian deconvolution approach (Sect. 3.3) was used to fit the model to observations by specifying a known ambient radon concentration. The markers show mean \pm one standard deviation.

choose to allow $p_{\mathrm{r}}$ to extend outside the range predicted by theory so as to achieve a good match with observations.

A further consequence of the simplicity of the model, and the nature of the test data, is that it can not be used to infer the reason for changes in $p_{r}$. Even though our data are consistent with a relative humidity effect, something which is also supported by previous studies (Frey et al., 1981; Su et al., 1988; Thomas and Leclare, 1970), these data can not rule out a temperature effect, because relative humidity is driven by temperature in these data. These issues are sidestepped during deconvolution by allowing $p_{\mathrm{r}}$ to take a random value within a range that encompasses the full range of observed detector responses. It is possible to take this approach because the variations in detector response are relatively small.

Variations in $p_{\mathrm{r}}$ imply that the net efficiency of the detector must also be changing. To measure variations in net efficiency, two changes were made. Instead of being closed between pulse injections, the source was flushed continuously so that a known concentration of radon could be injected each day. Instead of a 1 min injection, radon was injected for $1 \mathrm{~h}$ each day, for 32 consecutive days. The model was fit to observations to derive the net efficiency. The individual model parameters were not as well constrained as during the pulseinjection tests, so we discuss only the net efficiency derived from the $1 \mathrm{~h}$ injections.

In contrast to the apparent variation in $p_{\mathrm{r}}$, the net efficiency of the detector was more stable. This might be because the plateout rate, $\lambda_{\mathrm{p}}$, is also linked to diffusivity and will affect the net efficiency in the opposite sense to $p_{\mathrm{r}}$. The net efficiency had a coefficient of variation, ratio of standard deviation to mean, of 0.018 . On days where the relative humidity was below $15 \%$, however, the coefficient of variation ( 0.021$)$ was about double the remaining days (0.012). This degree of variability is comparable with the $0.02-0.06$ variability range of monthly calibrations across several sites (Chambers et al., 2015a).

Solar heating, as a cause of variations in sensitivity, was previously examined by Brunke et al. (2002). They found that the calibration coefficient of a $1500 \mathrm{~L}$ detector was more variable if the detector was unshaded, and attributed this to the effect of buoyancy-driven secondary circulations which increase the deposition rate of radon daughters within the delay chamber. However, they reported a coefficient of variation of 0.14 for the unshaded tests, almost seven times larger than our tests. The $700 \mathrm{~L}$ detector used here is also susceptible to secondary circulations, so it is likely that another factor was also important in the earlier results.

A possible explanation is the better temperature measurement in the newer version of the detector used in this study. In the present version, air temperature inside the delay chamber is measured using a dedicated sensor (Vaisala HMP45C), allowing the internal volumetric activity $\left(\mathrm{Bq} \mathrm{m}^{-3}\right)$ to be converted to ambient activity, or to equivalent activity at standard temperature and pressure $\left(20^{\circ} \mathrm{C}, 1000 \mathrm{hPa}\right.$, written $\mathrm{Bq} \mathrm{m}^{-3} \mathrm{STP}$ ). The Brunke et al. (2002) detector lacked an internal temperature sensor, relying instead on the temperature of a data logger for air density calculation. This data logger was inside the detector, but in an electronics chamber separate from the delay chamber. In our detector, the offset between the data logger temperature and the delay chamber temperature varies by $\pm 10^{\circ} \mathrm{C}$ from its mean value. Because the internal air density can differ from ambient conditions by $\sim 10 \%$, the air density correction can be nontrivial. An imperfect density correction might have been a contributor to the large variation in calibration coefficient, which was seen by Brunke et al. (2002) but not reproduced here.

In summary, the simplified model of the radon detector can be fit successfully to the measured detector response. By repeatedly measuring the detector response over several days, both the mean detector response and its variability can be established to a high degree of accuracy. Such knowledge of the detector response is necessary for getting good results from the deconvolution methods, described next.

\section{Deconvolution methods}

The task of deconvolution is to estimate a true signal from observations which are contaminated by noise and smeared by the non-ideal response of a detector. For a deconvolution technique to be applicable to radon measurements, it must handle Poisson-distributed noise, reconstruct a non-negative signal, and preserve the signal's long-term average. Ideally, it would also provide an uncertainty estimate and avoid the amplification of noise. 
Deconvolution can be expressed in terms of Bayesian parameter estimation (Gelman et al., 2013; MacKay, 2003). A key requirement is that, given a set of unobservable parameters, $\boldsymbol{\theta}$, a model is available to compute the likelihood of an observation vector, $\boldsymbol{y}$. The vector $\boldsymbol{\theta}$ contains parameters we want to know, i.e. the radon time series, as well as other parameters needed by the model to compute the detector response. The likelihood of observations $\boldsymbol{y}$, given parameter values $\boldsymbol{\theta}$, is written $p(\boldsymbol{y} \mid \boldsymbol{\theta})$. The quantity we require, the probability of $\boldsymbol{\theta}$ given actual observations $\boldsymbol{y}$, written $p(\boldsymbol{\theta} \mid \boldsymbol{y})$, is given by Bayes' rule

$p(\boldsymbol{\theta} \mid \boldsymbol{y})=\frac{p(\boldsymbol{\theta}) p(\boldsymbol{y} \mid \boldsymbol{\theta})}{p(\boldsymbol{y})}$,

meaning that, for a fixed set of observations $\boldsymbol{y}$,

$p(\boldsymbol{\theta} \mid \boldsymbol{y}) \propto p(\boldsymbol{\theta}) p(\boldsymbol{y} \mid \boldsymbol{\theta})$.

Therefore the task of computing the relative likelihood of $\boldsymbol{\theta}$, where each $\boldsymbol{\theta}$ is a sample from the posterior probability distribution, amounts to developing a model of the likelihood function, $p(\boldsymbol{y} \mid \boldsymbol{\theta})$, as well as assigning prior probabilities, $p(\boldsymbol{\theta})$, to the parameters.

For the radon detector, observed counts follow a Poisson distribution with a probability distribution given by

Poisson $(n \mid \lambda)=\frac{1}{n !} \lambda^{n} \exp (-\lambda)$,

where $n$ is the number of counts, an integer, and $\lambda$ is the expected value of the distribution. The expected value at time $t$ is given by the model, $\mathcal{M}(t \mid \boldsymbol{\theta})$, which was described in Sect. 2.2.

The net likelihood, $p(\boldsymbol{y} \mid \boldsymbol{\theta})$, is found from the product of the likelihood of the $N$ individual observations, namely

$p(\boldsymbol{y} \mid \boldsymbol{\theta})=\prod_{i=1}^{N} \operatorname{Poisson}\left(y_{i} \mid \mathcal{M}\left(t_{i} \mid \boldsymbol{\theta}\right)\right)$,

where $y_{i}$ is the number of counts observed in the $i$ th counting interval. We now consider three methods for making estimates of $\boldsymbol{\theta}$, and briefly describe the implementation.

\subsection{Richardson-Lucy deconvolution}

Richardson-Lucy (RL) deconvolution (Richardson, 1972; Lucy, 1974) is an iterative algorithm for image reconstruction, famously used for restoring images from the Hubble Space Telescope (Adorf et al., 1995). The RL method is a special case of the expectation-maximisation (EM) method (Dempster et al., 1977), derived for Poisson counting statistics. It follows from Eq. (12) under the assumption of a uniform distribution for the prior model parameters. In the solution, it is assumed that any non-negative value is equally likely.

A restriction of RL deconvolution is that the detector response must be known and unchanging. Should this be the case, $\mathcal{M}(t \mid \boldsymbol{\theta})$ can be written as a convolution of the pointresponse function, $s$, with the ambient radon concentration $\boldsymbol{\theta}$. Using $\otimes$ to signify the discrete convolution operator, the detector output is

$\boldsymbol{y}=\boldsymbol{\theta} \otimes s$,

where $\boldsymbol{y}, \boldsymbol{\theta}$, and $\boldsymbol{s}$ are discretised over the counting interval $\delta t$.

The maximum likelihood estimate, which maximises $p(\boldsymbol{y} \mid \boldsymbol{\theta})$, is found by iterating with the steps

$\boldsymbol{\theta}^{k+1}=\boldsymbol{\theta}^{k}\left(\frac{\boldsymbol{y}}{\boldsymbol{\theta}^{k} \otimes \boldsymbol{s}} \otimes \hat{\boldsymbol{s}}\right)$

where $\hat{\boldsymbol{s}}(t)=\boldsymbol{s}(-t)$ is the flipped point response function, $\boldsymbol{\theta}^{k}$ is the estimate at the $k$ th iteration, the initial guess is $\boldsymbol{\theta}^{0}=\boldsymbol{y}$, and division and multiplication are element-wise.

\subsection{Total variation regularisation for Richardson-Lucy deconvolution}

Without further refinement, RL deconvolution amplifies noise and leads to oscillatory solutions. Regularisation introduces an extra term into the optimisation which penalises oscillations and makes smooth solutions more likely (Dey et al., 2006; Dupé et al., 2012; Kempen and Vliet, 2000; Laasmaa et al., 2011).

One method is called total variation regularisation. Richardson-Lucy deconvolution with Total Variation regularisation (RL-TV) smooths the solution without reducing the sharpness of step changes (Rudin et al., 1992). With the introduction of an adjustable regularisation parameter, $\lambda_{\mathrm{TV}}$, the iteration step (Eq. 16) becomes (Dey et al., 2006)

$\boldsymbol{\theta}^{k+1}=\left(\frac{\boldsymbol{y}}{\boldsymbol{\theta}^{k} \otimes \boldsymbol{s}} \otimes \hat{\boldsymbol{s}}\right) \frac{\boldsymbol{\theta}^{k}}{1-\lambda_{\mathrm{TV}}\left|\frac{\mathrm{d} \boldsymbol{\theta}^{k}}{\mathrm{~d} t}\right|}$.

Increasing the size of $\lambda_{\mathrm{TV}}$ increases the amount of smoothing in the solution. A thorough examination of the effect of $\lambda_{\mathrm{TV}}$ is discussed by Kempen and Vliet (2000); we chose the value in an ad-hoc manner, using the smallest value which prevents oscillations during daytime periods when radon concentrations are steady.

Regularised Richardson-Lucy deconvolution has three important limitations which mean that it is not ideal for deconvolving the radon detector output. First, the pointresponse function must be known, whereas the radon detector response, being sensitive to environmental changes, is uncertain. Second, no uncertainty information is provided. Third, the regularisation parameter, which affects the final result, is chosen subjectively in our implementation. These limitations can be overcome using a Markov chain Monte-Carlo (MCMC) method which works directly with probability distributions. 


\subsection{Bayesian Monte-Carlo deconvolution}

This method, here termed Bayesian Monte-Carlo (BMC) deconvolution, is based on the general technique of Bayesian parameter estimation. A Markov chain is constructed, containing a large number of samples from the posterior probability function. Each sample is a possible realisation of the true time series, and other model parameters. Provided that the chain has been run for long enough to converge to the posterior distribution, an estimate of the time series statistics can be obtained directly from the Markov chain. Compared with the maximum-likelihood methods in the previous sections, generating the Markov chain is computationally expensive and can require tuning and supervision to ensure that convergence has been reached. Several features of our implementation were guided by the image reconstruction method of Esch et al. (2004).

In this method, the detector is modelled directly using the equations in Sect. 2.2. Prior beliefs are assigned to the detector parameters and the external radon concentration is represented as either a piecewise linear, or piecewise constant, function of time, depending on which is more appropriate to the problem at hand.

The radon concentration is strictly positive and a prior constraint is chosen which ensures that gradual changes in radon concentration are more likely than rapid changes. This constraint, based on the assumption that concentration changes arise from mixing processes and follow a lognormal distribution, is written

$\log C_{i} \sim \operatorname{Normal}\left(\mu=\log C_{i-1}, \sigma=\sigma_{\delta}\right)$,

where $C_{i}$ is the radon concentration at the $i$ th step and $\sigma_{\delta}$ is the width of the distribution. The distribution width has a physical meaning, it is equal to the standard deviation of $\log C_{i}-\log C_{i-1}$, and can be estimated from the variability of another tracer whose variability is likely to be similar to radon.

The detector parameters are drawn from normal distributions, which are wide enough to reproduce the observed changes in detector response. For the $700 \mathrm{~L}$ detector, these are

$$
\begin{aligned}
q_{\mathrm{e}} & \sim \operatorname{Normal}\left(\mu=40 \mathrm{Lmin}^{-1}, \sigma=0.02 \mu\right) \\
q & \sim \operatorname{Normal}\left(\mu=800 \mathrm{Lmin}^{-1}, \sigma=0.02 \mu\right) \\
\epsilon_{\mathrm{s}} & \sim \operatorname{Normal}(\mu=0.95, \sigma=0.05) \\
\log \lambda_{\mathrm{p}} & \sim \operatorname{Normal}(\mu=\log (1 / 180), \sigma=\log 2) .
\end{aligned}
$$

As well as the individual detector parameters, the net detector efficiency $\epsilon_{\text {net }}$ was constrained,

$\epsilon_{\text {net }} \sim \operatorname{Normal}(\mu=0.128, \sigma=0.05 \mu)$,

which is larger than the observed variability in $\epsilon_{\text {net }}(0.02$, Sect. 2.4) but matches the uncertainty in $\epsilon_{\text {net }}$ based on the calibration source. The constraint on $\epsilon_{\text {net }}$ means that samples with high values of $\epsilon_{\mathrm{s}}$, the screen capture efficiency, are more likely to have high values of the plateout constant $\lambda_{\mathrm{p}}$, so that $\epsilon_{\text {net }}$ stays within a reasonable range.

Samples are generated from the posterior distribution using the emcee sampler (Foreman-Mackey et al., 2013), an implementation of the affine invariant ensemble sampler of Goodman and Weare (2010). Other samplers considered were the NUTS sampler, implemented in Stan (Hoffman and Gelman, 2014), and PyMC (Patil et al., 2010). Our main reasons for choosing emcee were that it more easily accommodated our detector model than Stan, and implements a more sophisticated sampler than the current version of PyMC.

The emcee sampler works well when initialised around the maximum in probability space (Foreman-Mackey et al., 2013). In our implementation we use a two-stage approach to find this location. A first estimate is obtained using the regularised Richardson-Lucy method. This first estimate is then refined by using Powell's method (Powell, 1964; Press et al., 2007) to maximise Eq. (12). The estimate obtained is termed the maximum a posteriori probability (MAP) estimate. It differs from the maximum likelihood (ML) estimate by taking into account prior constraints on model parameters.

Because of the computational expense, and because the probability distribution is difficult to sample from, three numerical tricks are used to improve the sampler performance. First, the radon time series is broken into overlapping chunks. Shorter chunks, meaning fewer parameters, are easier to sample but each chunk must be longer than the response time of the detector. For the examples here, $24 \mathrm{~h}$ chunks with $6 \mathrm{~h}$ of overlap worked well.

A second useful trick was to transform the radon time series into a form inspired by the wavelet-like basis used by Esch et al. (2004), which reduces the correlation between parameters and makes the probability density easier to sample and optimise. The transformed parameters, $\phi_{i}$, were derived from the radon time series, $C_{i}$, according to

$$
\begin{aligned}
\phi_{1} & =\sum_{i=1}^{N} C_{i} \\
\phi_{i} & =\frac{C_{i}}{\phi_{1}-\sum_{j=2}^{i-1} C_{j}} .
\end{aligned}
$$

This transformation was more effective than the more common wavelet or Fourier transforms and had the most noticeable effect at the MAP optimisation step. The first parameter, $\phi_{1}$, can be thought of as the time integral of the signal and is strongly constrained by observations. The remaining $\phi_{2} \ldots \phi_{N}$ can each be interpreted as a fraction of the remaining integrated signal which the current point contributes.

The final trick, another transformation, was applied to bounded parameters. Parameters which were strictly positive, $\theta$, were transformed to unbounded parameters, $\phi$, ac- 
cording to

$\phi=\log \theta$

and parameters with upper, $b$, and lower, $a$, bounds were transformed using the logistic function

$\phi=\operatorname{logit}\left(\frac{\theta-a}{b-a}\right)$,

where

$\operatorname{logit}(x)=\log (x)-\log (1-x)$.

\section{Laboratory demonstration}

An initial test of the deconvolution methods was performed under controlled conditions, so that the deconvolution result could be compared with a known true signal. The known signal was generated by injecting a pulse of radon into the inlet for 1 hour at $770 \mathrm{~Bq} \mathrm{~m}^{-3}, \sim 10^{3}$ times ambient concentration, to generate a solitary square wave. Detector counts were recorded at $10 \mathrm{~min}$ intervals, and the deconvolution methods were used to reconstruct the input signal at $10 \mathrm{~min}$ resolution. Compared with atmospheric radon observations, such a change is unrealistically abrupt and poses a challenge to all of the deconvolution methods. On the other hand, the signalto-noise ratio during the injection period is much larger than typical atmospheric observations, and this makes deconvolution more precise.

A comparison between the three methods (Fig. 5) shows that all three are able to correctly redistribute the output signal into the 1 hour period when radon was being injected and to locate the edges of the square wave. There are also clear differences between the deconvolution methods. Without regularisation, Richardson-Lucy (RL) deconvolution produces oscillations in the solution, most visible in the detector background before and after the radon injection. Oscillations are completely suppressed during the peak with total variation regularisation (RL-TV), which favours step changes in the deconvolved signals, and background fluctuations are dramatically reduced.

After deconvolution, the radon time series is comparable to the output from a fast-response radon detector. The output from a RAD7, shown in Fig. 5c, is derived from the counts from the short-lived polonium-218 isotope, with a $3.05 \mathrm{~min}$ half-life.

Bayesian Monte Carlo (BMC) deconvolution produces a similar result to RL-TV, but the error estimate makes it clear that the reconstructed concentration fluctuations are not necessarily present during the injection period. The period from 14:00 to 16:00 LT is problematic, though. During this period, detector counts are slowly decaying from their peak count rate and the deconvolution output relies heavily on the accuracy of the model. The error bounds on the BMC deconvolution widen during this period, but not enough to include

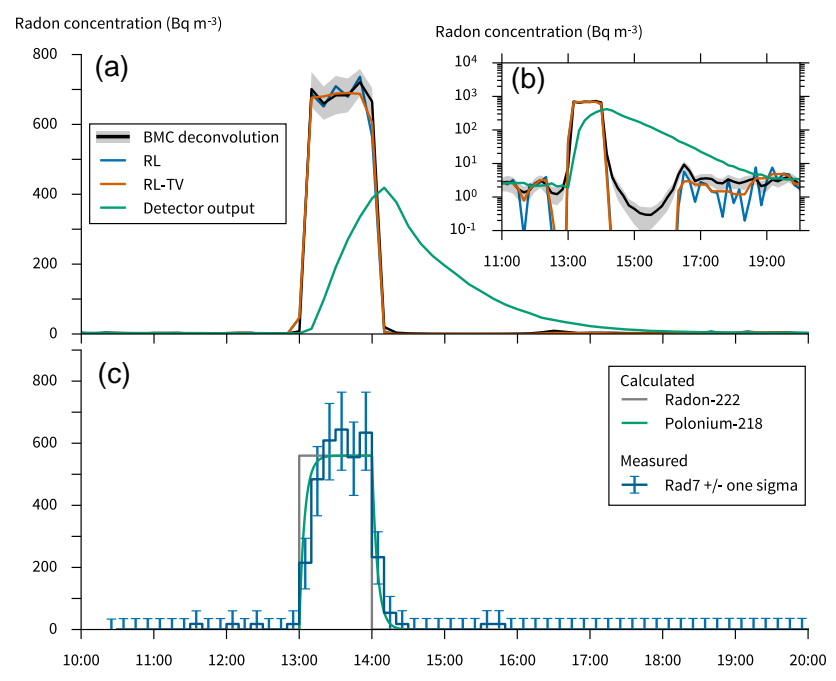

Figure 5. Observed counts and deconvolved output. An elevated concentration of radon was injected into the detector inlet between 13:00 and 14:00 LT with counts recorded at $10 \mathrm{~min}$ intervals. Observations were corrected for the detector response using the Bayesian Monte Carlo (BMC, with the 16th-84th percentile range shaded in grey), Richardson-Lucy (RL), and Richardson-Lucy with total variation regularisation (RL-TV) deconvolution methods. Panel (a) shows the output from the two-filter detector and the reconstructed time series. Panel (b) shows the same data on a log scale to emphasise the period of low radon concentrations before and after the spike. Panel (c) shows a repeat measurement where the twofilter detector has been substituted for a fast-response radon detector (RAD7, Durridge Company Inc.). The concentration of radon222 and polonium-218, computed from the source activity and flow rate, is also shown in (c).

the scenario of an abrupt drop back to a constant radon background, which is presumed to be the true signal, as supported by the RAD7 measurements. This reflects the prior constraint which favours smooth time series (Eq. 18), which is also the reason for the slower response of the BMC solution at 14:00 LT when compared with both RL and RL-TV.

The radon time series reconstruction is nevertheless a vast improvement over the scaled detector output. In this test, 10 min output is both feasible and realistic. The fact that prior beliefs are incorporated into the error limits needs to be kept in mind, however. Both the mean reconstruction and the error limits are influenced by the prior constraint which says that ambient radon concentrations are unlikely to change by a factor of $10^{3}$ over a $10 \mathrm{~min}$ period.

An obvious test, of the conclusion that the smoothness constraint is responsible for the low concentration, is to relax it or drop it entirely. It turns out, however, that it becomes difficult for the emcee sampler to draw samples from the posterior distribution in the absence of the smoothness constraint. It is not unexpected that this is a difficult distribution to sample. Distributions with a large number of parameters and non-linear models are problematic (Jasche and 
Wandelt, 2012), and the affine invariant sampler can fail on multi-modal distributions (Foreman-Mackey et al., 2013). It is possible that the smoothness constraint on the radon time series also smooths multi-modal peaks in the probability distribution.

The NUTS sampler, used by Stan, works well without the smoothness constraint. When applied to synthetic data, with a precisely known point-response function, uncertainty limits were indeed wider after the abrupt step back to ambient conditions (a plot is not shown). When applied to real data, however, small differences between the assumed and actual detector response caused ringing, or oscillations, in the output. Stan uses its own problem description language, making it difficult to incorporate the detector model necessary for a changing point response function, therefore the emcee sampler combined with a smoothness constraint is currently the best approach.

Although the smoothness constraint leads to subtle problems in this extreme case, it should improve the results for natural variations in radon concentration by preventing the amplification of noise.

\section{Reconstruction of simulated data}

Another test of our implementation of the Bayesian MonteCarlo method can be performed by simulating the detector response to a known time series and then applying our deconvolution algorithm. In this test, we know the "true" radon concentration precisely and can test the performance of the deconvolution algorithm in isolation. It tests the consistency of the method and also allows us to develop a feel for the link between model parameters, observational uncertainty, and the reconstructed time series.

The assumed radon concentration, shown in Fig. 6, is a simplified diurnal cycle based on the idealised near-surface radon concentration over land (Vinuesa et al., 2007). The radon concentration is constant during the daytime, and then increases at a constant rate during the night. The morning transition is modelled as an abrupt jump back to the daytime radon concentration. Like the laboratory test, the step-change back to daytime concentrations is unusually abrupt, so as to present a challenge to the deconvolution method.

The simulated radon detector output is derived from the response function of the $700 \mathrm{~L}$ radon detector with the addition of Poisson noise. As a result of its response time, the drop back to daytime radon concentrations takes about 3 hours.

In both Fig. 6a and b, the original time series is successfully reconstructed by the Bayesian Monte Carlo method. The assumed radon concentration is 10 times larger in Fig. 6a, peaking at $100 \mathrm{~Bq} \mathrm{~m}^{-3}$, which explains the smaller spread in concentration, relative to the peak size. Both examples represent realistic range in radon concentrations for inland or near-coastal sites.
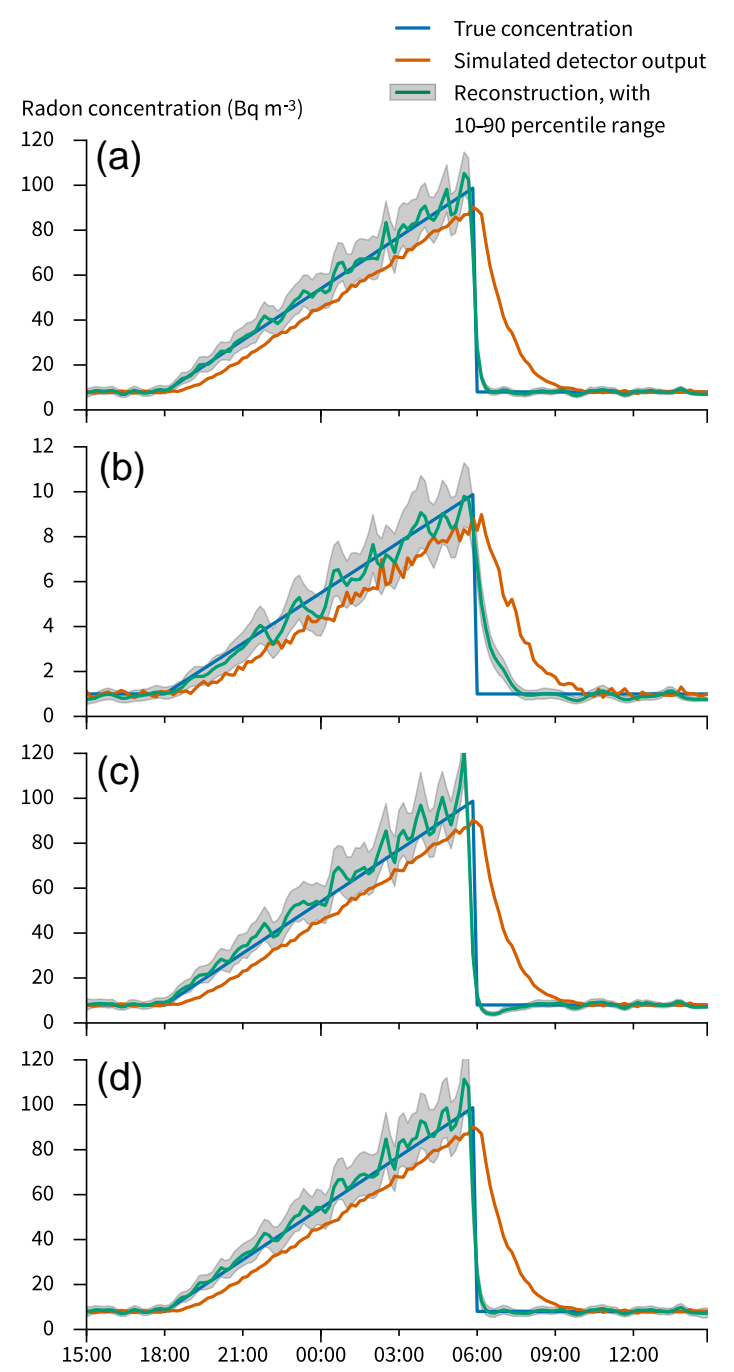

Figure 6. Tests of Bayesian Monte-Carlo deconvolution based on a simulation of the detector with known radon time series. Panel (a) shows a simulation with high radon concentrations, representative of an inland site on a stable night. In (b), the peak concentration is a factor of 10 lower. Panel (c) shows the effect of an incorrect prior, where the external flow rate was too low by a factor of two. Panel (d) has the same configuration as (c), but the sampler has run for ten times longer, $60 \times 10^{3}$ iterations.

In Fig. 6c, the prior constraint on $q_{\mathrm{e}}$ was deliberately set to an incorrect mean value, with a large uncertainty. That is,

$q_{\mathrm{e}} \sim \operatorname{Normal}\left(\mu=20 \mathrm{Lmin}^{-1}, \sigma=0.8 \mu\right)$,

half of the correct value $\left(\mu=40 \mathrm{Lmin}^{-1}\right.$ with $\left.\sigma=0.02 \mu\right)$. As shown in Fig. $3 \mathrm{a}, q_{\mathrm{e}}$ has a strong influence on the response function. Halving the external flow rate increases the response time, so that the deconvolution has a tendency to over-correct the time series.

After enough iterations, though, the deconvolution routine is able to compensate for the poorly specified prior and suc- 
cessfully reconstruct the true time series. While impressive in this case, it might be too optimistic to routinely expect performance to be as good in the real world, for at least three reasons. First, in a real detector, $q_{\mathrm{e}}$ is measured and is usually believed to be well known. If, for example, the flow sensor malfunctioned it seems likely that $q_{\mathrm{e}}$ would be wrongly specified, along with a narrow distribution. In this case, the algorithm would not be able to compensate. Second, the sampler takes much longer to reach convergence when $q_{\mathrm{e}}$ is poorly specified. In this example, it takes about 10 hours to run the required $60 \times 10^{3}$ iterations (on an Intel Core 2 Quad $\mathrm{CPU})$. Third, the radon concentrations are high in this example meaning that the signal-to-noise ratio is also high. This means that changing $q_{\mathrm{e}}$ has a significant effect on how well the model can be fit to observations.

For making routine corrections to observational data, fewer iterations would be used, that is $6 \times 10^{3}$ instead of $60 \times 10^{3}$, for faster processing. This is, in effect, an assumption that the priors are appropriate and the emcee sampler is initialised close to the probability-maximum in parameter space. As a result, it seems that inspection of the reconstructed time series is an important final step in applying the correction. Particular signs of problems are periods of oscillations or of tightly-bound low radon concentration after abrupt changes.

In summary, these simulations underline the importance of priors in the deconvolution process. In each of the cases, the most problematic period is the abrupt change back to daytime radon levels at 06:00 LT. This is when the smoothness constraint is violated in the time series. Although such abrupt changes are likely to be rare in observations, they are also likely to represent interesting events and therefore be studied more intently. In case $\mathrm{C}$, the incorrect specification of the external flow rate causes the radon concentration to undershoot the daytime value. This is because the external flow rate has a strong influence on the detector response function. In all of these cases, the radon concentration's uncertainty fails to grow enough to include the true value within the 10-90th percentile range, because the reconstructed estimate includes information from prior constraints. This is important to consider when making use of the reconstructed time series, because it implies that the reported uncertainty limits can not be relied upon as protection from inappropriate priors.

\section{Field application}

In this section, a case study approach is used to demonstrate the effect of deconvolution on radon time series and their interpretation. Radon observations can be used as both qualitative and quantitative tracers of vertical mixing either directly, or by comparison with another tracer emitted by the land surface. For the examples shown here, the deconvolved time series changes the interpretation when compared with the raw signal.

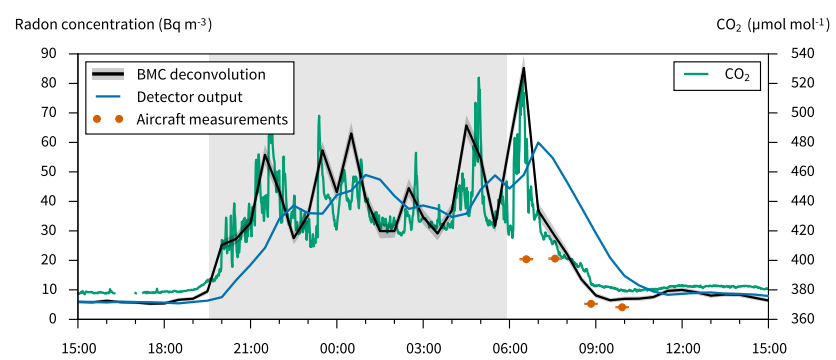

Figure 7. Field observations from a calm and clear night on the 5th-6th of November 2011. The wind speed was $\lesssim 1 \mathrm{~m} \mathrm{~s}^{-1}$ at $2 \mathrm{~m}$ a.g.l. for most of the night. Aircraft measurements were made $36-41 \mathrm{~m}$.a.g.l. over $6 \mathrm{~km}$ flight segments. Night-time is shaded grey, as is the 16th-84th percentile range about the reconstructed time series.

Prior to the characterisation experiments, the $700 \mathrm{~L}$ radon detector had been deployed in a 2-week field campaign alongside other sensors. The detector was outdoors, unshaded, and counts were recorded every $30 \mathrm{~min}$. In this section, we apply the Bayesian deconvolution method to these previously acquired measurements to see how the detector response affects interpretation of $30 \mathrm{~min}$ resolution data.

The study area was in an agricultural grazing region dominated by grassland and with gently sloping hills. The study area and ancillary measurements are described in detail by Williams et al. (2011). The radon detector was $\sim 500 \mathrm{~m}$ from a $10 \mathrm{~m}$ eddy-covariance and meteorological mast. Airborne radon measurements were taken from an ECO-Dimona motor glider equipped with activated charcoal filters. The filters were exposed during flight and analysed for radon content within $24 \mathrm{~h}$ (Williams et al., 2011). A closed path infra-red gas analyser (Li-7000, LI-COR inc.) measured $\mathrm{CO}_{2}$, with a 1 min averaging time, from a sampling tube co-located with the radon detector inlet. Soil radon emissions in the Goulburn area are comparable to the Australian average $\left(23.4 \pm 2 \mathrm{mBq} \mathrm{m}^{-2} \mathrm{~s}^{-1}\right)$, having previously been measured as $18.1 \mathrm{mBq} \mathrm{m}^{-2} \mathrm{~s}^{-1}$, during relatively wet conditions during winter, and $51.3 \mathrm{mBq} \mathrm{m}^{-2} \mathrm{~s}^{-1}$ in a dryer summer period (Griffiths et al., 2010).

Data from one night are presented in Fig. 7. Around sunset, both carbon dioxide and radon concentrations increase as the result of surface emissions being trapped within the stable nocturnal boundary layer. To assess the success of deconvolution, we compare radon with carbon dioxide fluctuations and assume that soil emissions are the only significant source of carbon dioxide. Following from this assumption, radon and carbon dioxide variations should both be driven mainly by atmospheric mixing and therefore be strongly correlated. Like radon, which is produced at a constant rate, the soil production of carbon dioxide should be relatively steady in this dry grassland (Kuzyakov and Gavrichkova, 2010). The presence of a reference time series is important because of the need to discriminate between real signal, which has been 
reconstructed, and spurious features in the time series caused by the amplification of noise.

Based on the reference carbon dioxide time series, deconvolution improves the radon observations in three ways. First, the time lag between carbon dioxide and radon is eliminated. Second, the night-time variability is increased which greatly improves the match with transient changes in tracer concentration during the night. Third, the apparent duration of the morning transition, from a stable to unstable boundary layer, is reduced in the reconstructed radon time series and now matches the time taken for carbon dioxide to return to daytime values.

Although the lag can also be greatly reduced by the much simpler means of removing a constant lag from the radon observations, a previously common practice, this will not change the time taken for radon concentrations drop during the morning transition. In the example shown, the detector counts take $4.5 \mathrm{~h}$ to drop to daytime values from their peak compared with $3 \mathrm{~h}$ for both carbon dioxide and the deconvolved radon time series. On days when the transition was more rapid (not shown), it was still $\sim 4.5 \mathrm{~h}$ before the detector output stabilised at day-time values.

As further evidence that the deconvolved observations are a better representation of the true radon concentration, there is a better agreement in the timing of the drop in radon concentration between the surface radon measurements and aircraft samples made $30 \mathrm{~m}$ overhead. The aircraft measurements are not directly comparable with the surface-based detector; the first two aircraft samples were made above the stable boundary-layer and all aircraft samples were made over $6 \mathrm{~km}$ flight segments. In contrast to the earlier samples, the later two were made within the comparably well mixed convective boundary layer and have a much smaller offset from the deconvolved time series. The remaining difference between the aircraft and fixed detector measurements is outside the limits of instrument uncertainty, but plausibly the result of spatial variability in the developing convective boundary layer.

When plotted against each other, carbon dioxide and radon are strongly related over almost 2 weeks of measurements. Focussing on a single night, Fig. 8a shows that they are linearly correlated and that deconvolution strengthens the correlation more than the removal of a constant $60 \mathrm{~min}$ lag, which is the optimum lag for maximising the correlation between radon and carbon dioxide (Fig. 8b). Deconvolution also corrects the slope of a linear fit to the points, changing it by $6 \%$ when compared with the constant-lag correction.

If the focus is only on the correlation, or ratio, of radon and carbon dioxide, it is possible to take the instrument response into account another way. Instead of deconvolving the radon observations, the response function of the radon detector can be applied to the carbon dioxide time series (Fig. 8c) also recovering a strong correlation between radon and carbon dioxide and the same slope.
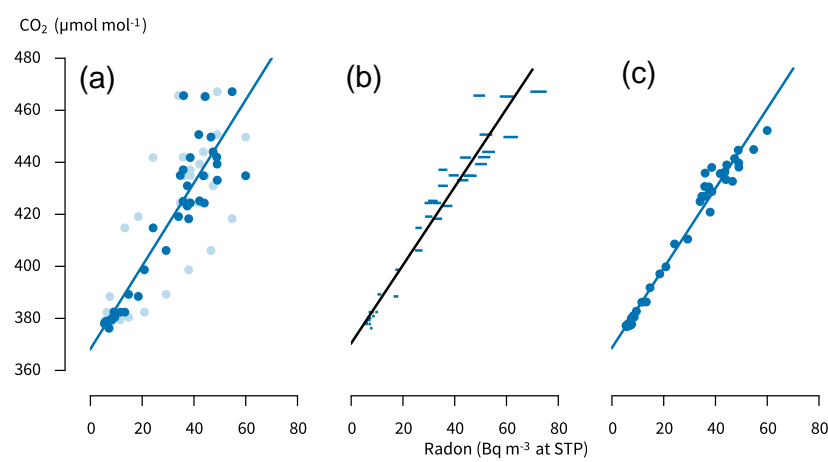

Figure 8. Correlation between radon and carbon dioxide concentrations during the same night as Fig. 7. In panel (a), raw light points indicate raw data and heavy points have the radon time series lagged by 1 hour (the linear fit is for the lagged points). In panel (b), radon measurements have been deconvolved to correct for the detector response and the horizontal bars show the range between 10th and 90th percentiles. Panel (c) shows the raw radon measurements versus carbon dioxide concentrations which have been filtered with a running mean filter weighted by the radon detector response. The slopes of the three linear fits are (a): 1.60, (b): $1.50 \pm 0.07$, (c): $1.53\left(\right.$ umol mol$\left.^{-1}\right) /\left(\mathrm{Bqm}^{-3} \mathrm{STP}\right)$.

During a single night the slope of radon versus carbon dioxide is related to the flux ratios. Assuming that the net respiration flux of carbon dioxide is constant during the night, and that radon and carbon dioxide have the same source distribution in space, the fluxes, $F_{\mathrm{CO}_{2}}$ and $F_{\mathrm{Rn}}$, can be linked to the change in concentration since sunset, $\Delta C_{\mathrm{CO}_{2}}$ and $\Delta C_{\mathrm{Rn}}$, according to (e.g. Conen et al., 2002)

$\frac{F_{\mathrm{Rn}}}{F_{\mathrm{CO}_{2}}}=\frac{\Delta C_{\mathrm{Rn}}}{\Delta C_{\mathrm{CO}_{2}}}$

As an extension, beyond correcting for the instrument response, it would also be useful to examine deviations from a linear correlation between radon and carbon dioxide. These deviations, which are relatively small at this site, would be caused by differences in the spatial variability of sources (van der Laan et al., 2014), or by temporal changes in carbon dioxide emissions. For this paper, though, it is pursued no further.

Two nights earlier, during a windy night, the respiration flux from eddy covariance measurements was $F_{\mathrm{CO}_{2}}=$ $1.18 \mu \mathrm{mol} \mathrm{m}^{-2} \mathrm{~s}^{-1}$. Assuming that the respiration flux was similar on the calm night, when eddy covariance estimates are known to be inaccurate (Aubinet, 2008; Mahrt, 2010), the radon flux was $F_{\mathrm{Rn}}=19.2_{-0.9}^{+0.8} \mathrm{mBq} \mathrm{m}^{-2} \mathrm{~s}^{-1}$. This estimate is within the previously measured regional emissions (Griffiths et al., 2010) of 18.1 and $51.3 \mathrm{mBq} \mathrm{m}^{-2} \mathrm{~s}^{-1}$ and has an uncertainty derived from the uncertainty in $\Delta C_{\mathrm{Rn}} / \Delta C_{\mathrm{CO}_{2}}$, ignoring the uncertainty in the eddy-covariance flux and systematic errors.

Having obtained an estimate of the radon flux, it is possible to estimate the depth of the planetary boundary layer 


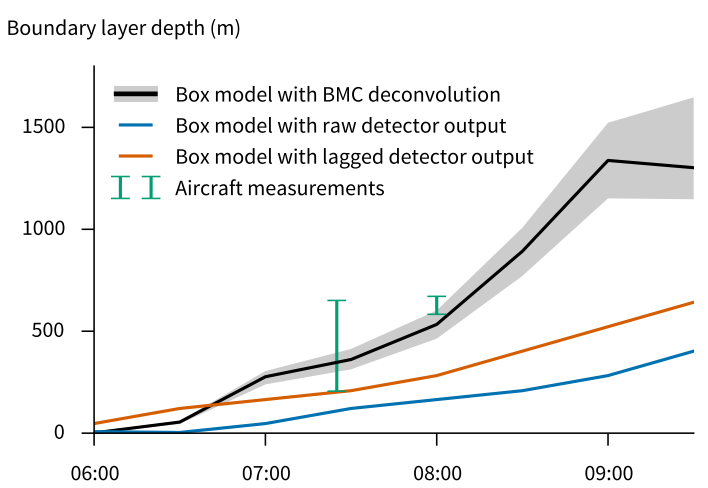

Figure 9. Box model analysis of deconvolved and scaled radon detector output. The 16th-84th percentile range is shaded grey. The mixing depth is calculated by assuming that radon is mixed homogeneously in a near-surface layer, with surface emissions of $F_{\mathrm{Rn}}=19.2 \mathrm{mBq} \mathrm{m}^{-2} \mathrm{~s}^{-1}$. The aircraft measurements of boundarylayer depth are computed from vertical profiles of temperature, wind speed, and humidity using the parcel method (lower limit of each bar) and bulk Richardson number methods (the upper limit of each bar). A critical Richardson number of 0.3 was assumed.

based on the radon time series and a boundary layer budget. During the morning transition, the boundary layer is likely to be well mixed so that a simple box model (Griffiths et al., 2013) can be used to estimate its depth, on the assumption that the horizontal flow is non-divergent. Output from this box model is shown in Fig. 9 based on raw, 60 min lagged, and deconvolved time series. The motor glider flew ascending profiles at 07:25 and 08:00 LT and profiles of temperature, humidity, and wind speed were used to derive boundary layer heights. The aircraft-derived boundary layer height was computed from two methods: the parcel method (Collaud Coen et al., 2014), which has similar underlying assumptions to the radon box model, and a bulk Richardson number method (Holtslag and Boville, 1993).

In Fig. 9, the profile flown at 08:00 LT was capped by a strong temperature inversion, which draws the parcel and Richardson number estimates closer than the earlier profile. As well as being easier to analyse, a strongly capped boundary layer is close to the situation assumed by the box model. As a result, the box-model-derived height agrees well with the aircraft measurements at 08:00 LT, and is in between the two aircraft-derived estimates at 07:25, provided that the deconvolved radon time series is used. Using the detector output directly leads to an underestimate of mixing height. Alternatively, if the radon surface flux were a free parameter, surface emissions would be overestimated. In this case, using the scaled detector output yields a mixing height estimate which is too low by a factor of four. If the detector output is lagged by a constant $60 \mathrm{~min}$, the error is reduced, yet the boundary layer height is still low by a factor of two and the timing of boundary layer growth is wrong.

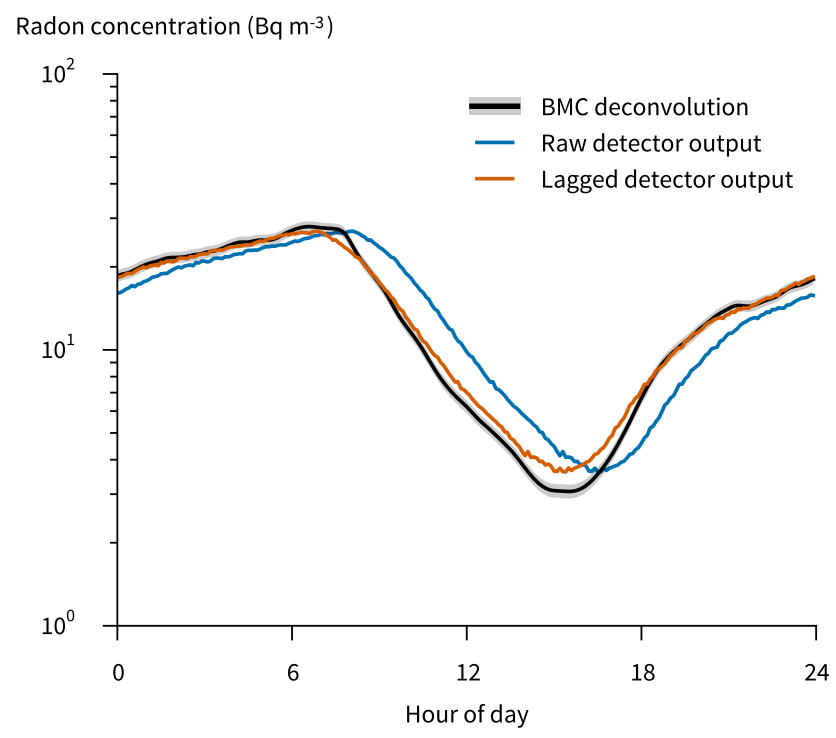

Figure 10. Composite diurnal cycle from the Richmond $1500 \mathrm{~L}$ detector during 2 weeks of consecutive stable nights and clear-sky days. BMC deconvolution means the Bayesian Monte Carlo deconvolution method from Sect. 3.3 and the 16th-84th percentile range is shaded grey. A lag correction of 72 minutes maximises the correlation between the lagged detector output and the deconvolved output. See Chambers et al. (2015b) for site details.

In this case (Fig. 7) the raw and deconvolved time series are in agreement during the afternoon. It is important to know if this is always the case, because the daytime radon concentration is of particular interest during model testing (e.g. Allen et al., 1996). Daytime radon concentrations respond to the height of the convective boundary layer and airmass fetch (Chambers et al., 2011). Errors in daytime radon concentrations might therefore be related to problems with the modelled convective boundary layer and not to factors which are important for night-time radon concentrations.

To see how the detector response may influence model comparisons, the systematic influence of deconvolution on daytime radon concentrations was examined for a 2-week period of radon measurements from a site near Richmond, New South Wales (Chambers et al., 2015b). Figure 10 shows the composite diurnal cycle from a $1500 \mathrm{~L}$ detector during 2 weeks of clear weather conditions, when the radon diurnal amplitude was large, meaning that the influence of the detector response might be relatively large. In this case, the detector recorded counts every $6 \mathrm{~min}$ and a lag correction of 72 min maximises the correlation between raw and deconvolved time series. A $60 \mathrm{~min}$ lag works almost as well, and would be a good choice for data recorded at the usual $30 \mathrm{~min}$ intervals. The constant-lag correction reduces the daytime high bias present in the raw detector output, but changes the timing of the morning drop in radon concentration. Crucially, for comparison with model output, the afternoon high bias is still present (18\% high both at 15:00 LT and in the daily min- 
imum). This is significantly higher than the absolute uncertainty in the detector at steady state (a $5 \%$ uncertainty, based on the uncertainty of the calibration source).

In summary, the two case studies in this section illustrated that correcting for the instrument response can lead to relatively large changes in the interpretation of radon time series. The first showed that raw radon time series are inconsistent with carbon dioxide measurements, with grab samples, and with aircraft profiles of the boundary-layer. After deconvolution, however, the time series is consistent with a uncomplicated understanding of boundary-layer mixing and carbon dioxide fluxes. The second showed the effect of deconvolution on the radon diurnal composite. The effect is less dramatic than the previous example, but significant because it biases results. The amplitude of the diurnal cycle is attenuated in the raw radon time series causing a high bias of $\sim 18 \%$ in the daily minimum. This high bias can be expected at other inland sites during periods of clear skies and low winds.

\section{Discussion}

\subsection{Detector operation}

The deconvolution technique performs best with a stable detector response function. Section 2.4 showed that a detector being operated outdoors has a small, but measurable, change in response which appears to be linked to low relative humidity, itself caused by the hotter-than-ambient temperature inside the delay chamber. The detrimental effect of solar heating has been observed elsewhere; Brunke et al. (2002) found that shading their detector led to more repeatable calibrations of detector efficiency. For best performance, therefore, indoor installation should be favoured. Actively controlling the humidity inside the detector is also a possibility for future consideration. If the detector response were stabilised enough, it would simplify the detector model and make deconvolution easier. The long-tail response of the detector is ultimately set by the lifetimes of radon daughters but the initial rise-time is controlled by the external flow rate. Provided that thoron can be excluded from the detector, a higher external flow rate should be favoured when aiming for sub-hourly resolution.

\subsection{Application to other two-filter radon detectors}

Analysis of the radon detector response and deconvolution methods has focused mainly on data from a single instrument. Because the instrument response depends on parameters which are not precisely standardised, the correction can not be applied directly to other installations. Nevertheless, it is possible to derive the response of other detectors without further characterisation experiments because of the routine calibrations which are performed during operation and because many of the detector parameters are recorded.
Routine data acquisition uses 30 min counting intervals. Each month, for calibration, a solenoid valve opens to allows radon emitted from a calibration source to enter the main delay chamber of the detector. The calibration period typically lasts several hours so that the detector can approach steady state. Then the valve closes, and the detector returns to measuring ambient air. By fitting the model to the calibration data, we are able to derive the detector response from the shape of the calibration peak. The detector response, found using this method, is not as well-constrained as the detector response we measured using a 1 min injection and the effect of any upstream delay chambers is not measured. For the best possible results, especially at 10 min resolution, it would be appropriate to measure the detector response. Even so, the detector response, derived from calibration, is good enough to reconstruct $30 \mathrm{~min}$ or hourly measurements from archived data.

\subsection{When should deconvolution be used?}

Three deconvolution methods were tested in this work, all of which are successful at redistributing the long response tail into the correct part of the time series. Nevertheless, deconvolution is not always the right option.

Deconvolution introduces more variability into the time series. For the method used here, unrealistic fluctuations are suppressed by a prior constraint on the radon time series. Deviations from a smooth signal are only allowed when there is enough evidence, from detector counts, to support them. When detector counts, $N$, are small measurement noise is large because noise scales with $\sqrt{N}$. At remote baseline sites, radon concentrations of $50 \mathrm{mBq} \mathrm{m}^{-3}$ are common (Zahorowski et al., 2013), 3 orders of magnitude below the radon concentrations in Fig. 7. During these periods, the case for deconvolution is weak.

Even in cases where deconvolution is possible, it might not be necessary. When the high-frequency fluctuations are not of intrinsic interest, there are two alternatives. First, a constant lag can be assumed, as has been standard practice previously. Second, in cases where radon measurements are compared with another tracer, the detector response can be applied to the other time series.

The constant-lag approach has the advantage of simplicity and can be useful in many studies. The choice of lag might be based on some characteristic of the instrument response, or chosen to maximise the correlation with other tracers (e.g. Griffiths et al., 2014). With a constant-lag approach, the correlation between radon and other tracers, although weakened, is still present. There are also biases which affect the diurnal cycle, but at least these can be anticipated.

Applying the detector response to other tracers is also simpler than deconvolution. It requires the detector response to be well understood, but the complexities of Markov chain Monte Carlo sampling can be avoided. For the case study discussed here, applying the detector response to carbon diox- 
ide measurements is a feasible option. As this is, in effect, smoothing the carbon dioxide measurements, this approach reduces the statistical uncertainty without changing the abundance ratio of the two species.

A forward modelling approach could also be attractive for model-data intercomparison, but would require care during interpretation. Problems could arise under certain conditions, such as when trying to compare daytime model and measured radon concentrations. Section 6, showed that the daytime radon measurements retain a memory of the night-time peak, with the implication that a diagnosed daytime bias might be caused by errors in night-time mixing.

In spite of the possibility of forward-modelling, deconvolution remains preferable in many cases, especially when radon concentrations are used quantitatively. An example is when comparing with grab samples or with widely spaced model output. In particular, deconvolution is the best option when radon measurements are used to infer the depth of boundary-layer mixing.

\section{Conclusions}

The raw signal from a two-filter radon detector responds slowly to abrupt changes in radon concentration. The signal, as a result, depends on the history of radon concentration for several hours prior. Correcting for the instrument response, or deconvolving the radon time series, is difficult because of small day-to-day changes in the instrument response. Nevertheless, this paper shows that it is possible to reconstruct the ambient radon time series using a Bayesian deconvolution method based on a Markov chain Monte Carlo sampler and a validated model of the detector response.

For archived measurements, the radon time series was reconstructed at $30 \mathrm{~min}$ resolution, the resolution of the raw data, instead of being down-sampled to hourly resolution. For large radon signals, such as from a calibration source, $10 \mathrm{~min}$ resolution was achievable and could potentially be applied to field measurements inland, where the radon concentration is high.

When sub-diurnal measurements of radon concentration are studied, deconvolution can change their interpretation. This is especially the case at inland sites with large variations in the boundary-layer height under clear sky conditions. In one case study, the deconvolved time series was consistent with carbon dioxide measurements (from an instrument with a fast time response), grab samples, and a simple mixing height model; the uncorrected detector output was not. In another example, during a period with a large amplitude diurnal cycle, the mean daily minimum radon concentration was too high by $18 \%$, if the instrument response was neglected.

In terms of physical properties radon is an almost ideal natural tracer, which makes it important to account for the non-ideal characteristics of detectors. Correcting for the instrument response, based on the representative cases shown here, makes inference from radon observations easier and more certain.

\section{The Supplement related to this article is available online at doi:10.5194/amt-9-2689-2016-supplement.}

Acknowledgements. We thank Ot Sisoutham, Adrian Element, and Wlodek Zahorowski for their roles in the construction and operation of the two-filter radon detectors used in this study, Jagoda Crawford and Sander van der Laan for helpful comments on a draft version of the manuscript, Airborne Research Australia for aircraft measurements, Dioni Cendon loaning us a RAD7, and ANSTO Scientific Computing for supporting access to their high performance computing facility. We also acknowledge the authors of open-source software used in this study, especially the Python scientific computing stack (including numpy, scipy, matplotlib, pandas).

Edited by: D. Brunner

\section{References}

Adorf, H.-M., Hook, R. N., and Lucy, L. B.: HST image restoration developments at the ST-ECF, Int. J. Imaging Syst. Technol., 6, 339-349, doi:10.1002/ima.1850060407, 1995.

Ahnert, K. and Mulansky, M.: Odeint - solving ordinary differential equations in $\mathrm{C}++$, in: AIP Conference Proceedings, AIP Publishing, vol. 1389, 1586-1589, doi:10.1063/1.3637934, 2011.

Allen, D. J., Rood, R. B., Thompson, A. M., and Hudson, R. D.: Three-dimensional radon 222 calculations using assimilated meteorological data and a convective mixing algorithm, J. Geophys. Res., 101, 6871-6881, doi:10.1029/95JD03408, 1996.

Alonso, M., Kousaka, Y., Hashimoto, T., and Hashimoto, N.: Penetration of nanometer-sized aerosol particles through wire screen and laminar flow tube, Aerosol Sci. Technol., 27, 471-480, doi:10.1080/02786829708965487, 1997.

Aubinet, M.: Eddy covariance $\mathrm{CO}_{2}$ flux measurements in nocturnal conditions: an analysis of the problem, Ecol. Appl., 18, 13681378, doi:10.1890/06-1336.1, 2008.

Biraud, S., Ciais, P., Ramonet, M., Simmonds, P., Kazan, V., Monfray, P., O’Doherty, S., Spain, T. G., and Jennings, S. G.: European greenhouse gas emissions estimated from continuous atmospheric measurements and radon 222 at Mace Head, Ireland, J. Geophys. Res., 105, 1351-1366, doi:10.1029/1999JD900821, 2000.

Brunke, E. G., Labuschagne, C., Parker, B., van der Spuy, D., and Whittlestone, S.: Cape Point GAW Station ${ }^{222}$ Rn detector: factors affecting sensitivity and accuracy, Atmos. Environ., 36, 2257-2262, doi:10.1016/S1352-2310(02)00196-6, 2002.

Brunke, E.-G., Labuschagne, C., Parker, B., Scheel, H., and Whittlestone, S.: Baseline air mass selection at Cape Point, South Africa: application of ${ }^{222} \mathrm{Rn}$ and other filter criteria to $\mathrm{CO}_{2}$, Atmos. Environ., 38, 5693-5702, doi:10.1016/j.atmosenv.2004.04.024, 2004. 
Chambers, S., Williams, A. G., Zahorowski, W., Griffiths, A., and Crawford, J.: Separating remote fetch and local mixing influences on vertical radon measurements in the lower atmosphere, Tellus B, 63, 843-859, doi:10.1111/j.1600-0889.2011.00565.x, 2011.

Chambers, S. D., Hong, S.-B., Williams, A. G., Crawford, J., Griffiths, A. D., and Park, S.-J.: Characterising terrestrial influences on Antarctic air masses using Radon-222 measurements at King George Island, Atmos. Chem. Phys., 14, 9903-9916, doi:10.5194/acp-14-9903-2014, 2014.

Chambers, S. D., Williams, A. G., Conen, F., Griffiths, A. D., Riemann, S., Steinbacher, M., Krummel, P., Steele, L., van der Schoot, M. V., Galbally, I., Molloy, S. B., and Barnes, J.: Towards a universal "baseline" characterisation of air masses for highand low-altitude observing stations using radon-222, Aerosol Air Qual. Res., doi:10.4209/aaqr.2015.06.0391, 2015a.

Chambers, S. D., Williams, A. G., Crawford, J., and Griffiths, A. D.: On the use of radon for quantifying the effects of atmospheric stability on urban emissions, Atmos. Chem. Phys., 15, 1175-1190, doi:10.5194/acp-15-1175-2015, 2015 b.

Cheng, Y. S. and Yeh, H. C.: Theory of a screen-type diffusion battery, J. Aerosol Sci., 11, 313-320, doi:10.1016/00218502(80)90105-6, 1980.

Cheng, Y. S., Keating, J. A., and Kanapilly, G. M.: Theory and calibration of a screen-type diffusion battery, J. Aerosol Sci., 11, 549-556, doi:10.1016/0021-8502(80)90127-5, 1980.

Collaud Coen, M., Praz, C., Haefele, A., Ruffieux, D., Kaufmann, P., and Calpini, B.: Determination and climatology of the planetary boundary layer height above the Swiss plateau by in situ and remote sensing measurements as well as by the COSMO-2 model, Atmos. Chem. Phys., 14, 13205-13221, doi:10.5194/acp14-13205-2014, 2014.

Conen, F. and Robertson, L. B.: Latitudinal distribution of radon-222 flux from continents, Tellus B, 54, 127-133, doi:10.1034/j.1600-0889.2002.00365.x, 2002.

Conen, F., Neftel, A., Schmid, M., and Lehmann, B. E.: $\mathrm{N}_{2} \mathrm{O} /{ }^{222} \mathrm{Rn}$ - soil flux calibration in the stable nocturnal surface layer, Geophys. Res. Lett., 29, 1025, doi:10.1029/2001GL013429, 2002.

Considine, D. B., Bergmann, D. J., and Liu, H.: Sensitivity of Global Modeling Initiative chemistry and transport model simulations of radon-222 and lead-210 to input meteorological data, Atmos. Chem. Phys., 5, 3389-3406, doi:10.5194/acp-5-33892005, 2005.

Dankelmann, V., Reineking, A., and Postendörfer, J.: Determination of neutralisation rates of ${ }^{218}$ Po ions in air, Radiat. Prot. Dosim., 94, 353-357, http://rpd.oxfordjournals.org/content/94/4/ 353,2001

Dempster, A. P., Laird, N. M., and Rubin, D. B.: Maximum likelihood from incomplete data via the EM algorithm, J. R. Stat. Soc. Series B Stat. Methodol., 39, 1-38, http://www.jstor.org/stable/ 2984875, 1977.

Dey, N., Blanc-Feraud, L., Zimmer, C., Roux, P., Kam, Z., Olivo-Marin, J.-C., and Zerubia, J.: Richardson-Lucy algorithm with total variation regularization for $3 \mathrm{D}$ confocal microscope deconvolution, Microsc. Res. Tech., 69, 260-266, doi:10.1002/jemt.20294, 2006.

Dupé, F. X., Fadili, M. J., and Starck, J. L.: Deconvolution under Poisson noise using exact data fidelity and syn- thesis or analysis sparsity priors, Stat. Methodol., 9, 4-18, doi:10.1016/j.stamet.2011.04.008, 2012.

Ehrlich, A. and Wendisch, M.: Reconstruction of high-resolution time series from slow-response broadband terrestrial irradiance measurements by deconvolution, Atmos. Meas. Tech., 8, 36713684, doi:10.5194/amt-8-3671-2015, 2015.

Esch, D. N., Connors, A., Karovska, M., and van Dyk, D. A.: An image restoration technique with error estimates, Astrophys. J., 610, 1213-1227, doi:10.1086/421761, 2004.

Foreman-Mackey, D., Hogg, D. W., Lang, D., and Goodman, J.: emcee: The MCMC Hammer, Publ. Astron. Soc. Pac., 125, 306312, doi:10.1086/670067, 2013.

Frank, G., Steinkopff, T., and Salvamoser, J.: Low Level Measurement of ${ }^{222} \mathrm{Rn}$ in the Atmosphere in the Frame of the Global Atmospheric Watch Programme, in: Sources and Measurements of Radon and Radon Progeny Applied to Climate and Air Quality Studies, IAEA Proceedings Series, p. 105, IAEA, Vienna, 2012.

Frey, G., Hopke, P. K., and Stukel, J. J.: Effects of trace gases and water vapor on the diffusion coefficient of polonium-218, Science, 211, 480-481, doi:10.1126/science.211.4481.480, 1981.

Gäggeler, H., Jost, D., Baltensperger, U., Schwikowski, M., and Seibert, P.: Radon and thoron decay product and ${ }^{210} \mathrm{~Pb}$ measurements at Jungfraujoch, Switzerland, Atmos. Environ., 29, 607616, doi:10.1016/1352-2310(94)00195-Q, 1995.

Gelman, A., Carlin, J. B., Stern, H. S., Dunson, D. B., Vehtari, A., and Rubin, D. B.: Bayesian data analysis, vol. 2, Chapman and Hall, 2013.

Goodman, J. and Weare, J.: Ensemble samplers with affine invariance, Comm. App. Math. Comp. Sci., 5, 65-80, doi:10.2140/camcos.2010.5.65, 2010.

Griffiths, A. D., Zahorowski, W., Element, A., and Werczynski, S.: A map of radon flux at the Australian land surface, Atmos. Chem. Phys., 10, 8969-8982, doi:10.5194/acp-10-8969-2010, 2010.

Griffiths, A. D., Parkes, S. D., Chambers, S. D., McCabe, M. F., and Williams, A. G.: Improved mixing height monitoring through a combination of lidar and radon measurements, Atmos. Meas. Tech., 6, 207-218, doi:10.5194/amt-6-207-2013, 2013.

Griffiths, A. D., Conen, F., Weingartner, E., Zimmermann, L., Chambers, S. D., Williams, A. G., and Steinbacher, M.: Surfaceto-mountaintop transport characterised by radon observations at the Jungfraujoch, Atmos. Chem. Phys., 14, 12763-12779, doi:10.5194/acp-14-12763-2014, 2014.

Grossi, C., Vargas, A., Camacho, A., López-Coto, I., Bolívar, J., Xia, Y., and Conen, F.: Inter-comparison of different direct and indirect methods to determine radon flux from soil, Radiat. Meas., 46, 112-118, 2011.

Grossi, C., Arnold, D., Adame, J., López-Coto, I., Bolívar, J., de la Morena, B., and Vargas, A.: Atmospheric ${ }^{222} \mathrm{Rn}$ concentration and source term at El Arenosillo $100 \mathrm{~m}$ meteorological tower in southwest Spain, Radiat. Meas., 47, 149-162, doi:10.1016/j.radmeas.2011.11.006, 2012.

Guedalia, D., Lopez, A., Fontan, J., and Birot, A.: Aircraft measurements of Rn-222, Aitken nuclei and small ions up to $6 \mathrm{~km}$, J. Appl. Meteorol., 11, 357-365, doi:10.1175/15200450(1972)011<0357:AMORAN>2.0.CO;2, 1972.

Heim, M., Mullins, B. J., Wild, M., Meyer, J., and Kasper, G.: Filtration Efficiency of Aerosol Particles Below 20 Nanometers, Aerosol Sci. Technol., 39, 782-789, doi:10.1080/02786820500227373, 2005. 
Heim, M., Attoui, M., and Kasper, G.: The efficiency of diffusional particle collection onto wire grids in the mobility equivalent size range of $1.2-8 \mathrm{~nm}$, J. Aerosol Sci., 41, 207-222, doi:10.1016/j.jaerosci.2009.10.002, 2010.

Hoffman, M. D. and Gelman, A.: The no-U-turn sampler: Adaptively setting path lengths in Hamiltonian Monte Carlo, J. Mach. Learn. Res., 15, 1593-1623, http://jmlr.org/papers/v15/ hoffman14a.html, 2014.

Holtslag, A. and Boville, B.: Local versus nonlocal boundary-layer diffusion in a global climate model, J. Clim., 6, 1825-1842, doi:10.1175/1520-0442(1993)006<1825:LVNBLD>2.0.CO;2, 1993.

Ichitsubo, H., Hashimoto, T., Alonso, M., and Kousaka, Y.: Penetration of ultrafine particles and ion clusters through wire screens, Aerosol Sci. Technol., 24, 119-127, doi:10.1080/02786829608965357, 1996.

Jacob, D. J. and Prather, M. J.: Radon-222 as a test of convective transport in a general circulation model, Tellus B, 42, 118-134, doi:10.1034/j.1600-0889.1990.00012.x, 1990.

Jasche, J. and Wandelt, B. D.: Bayesian inference from photometric redshift surveys, Mon. Not. R. Astron. Soc., 425, 1042-1056, doi:10.1111/j.1365-2966.2012.21423.x, 2012.

Jonassen, N. and McLaughlin, J. P.: On the recoil of RaB from membrane filters, J. Aerosol Sci., 7, 141-149, doi:10.1016/00218502(76)90070-7, 1976.

Karstens, U., Schwingshackl, C., Schmithüsen, D., and Levin, I.: A process-based ${ }^{222}$ radon flux map for Europe and its comparison to long-term observations, Atmos. Chem. Phys., 15, 1284512865, doi:10.5194/acp-15-12845-2015, 2015.

Kempen, V. and Vliet, V.: The influence of the regularization parameter and the first estimate on the performance of Tikhonov regularized non-linear image restoration algorithms, J. Microsc., 198, 63-75, doi:10.1046/j.1365-2818.2000.00671.x, 2000.

Knutson, E. and George, A.: Measurements of ${ }^{214} \mathrm{~Pb}$ loss by recoil from decay of ${ }^{218}$ Po collected on a wire screen, Abstracts of the 1994 European Aerosol Conference, 25, Supplement 1, 71-72, doi:10.1016/0021-8502(94)90266-6, 1994.

Kuzyakov, Y. and Gavrichkova, O.: REVIEW: Time lag between photosynthesis and carbon dioxide efflux from soil: a review of mechanisms and controls, Glob. Chang. Biol., 16, 3386-3406, doi:10.1111/j.1365-2486.2010.02179.x, 2010.

Laasmaa, M., Vendelin, M., and Peterson, P.: Application of regularized Richardson-Lucy algorithm for deconvolution of confocal microscopy images, J. Microsc., 243, 124-140, doi:10.1111/j.1365-2818.2011.03486.x, 2011.

Levin, I., Born, M., Cuntz, M., Langendörfer, U., Mantsch, S., Naegler, T., Schmidt, M., Varlagin, A., Verclas, S., and Wagenbach, D.: Observations of atmospheric variability and soil exhalation rate of radon-222 at a Russian forest site - Technical approach and deployment for boundary layer studies, Tellus B, 54, 462475, doi:10.1034/j.1600-0889.2002.01346.x, 2002.

Lucy, L. B.: An iterative technique for the rectification of observed distributions, Astron. J., 79, 745, doi:10.1086/111605, 1974.

MacKay, D. J.: Information theory, inference, and learning algorithms, Cambridge University Press, 7th Edn., available at: http: //www.inference.phy.cam.ac.uk/mackay/itila/, 2003.

Mahrt, L.: Computing turbulent fluxes near the surface: Needed improvements, Agric. For. Meteorol., 150, 501-509, doi:10.1016/j.agrformet.2010.01.015, 2010.
Martin, P., Tims, S., Ryan, B., and Bollhöfer, A.: A radon and meteorological measurement network for the Alligator Rivers Region, Australia, J. Environ. Radioact., 76, 35-49, doi:10.1016/j.jenvrad.2004.03.017, 2004.

Massman, W. J.: A simple method for estimating frequency response corrections for eddy covariance systems, Agric. For. Meteorol., 104, 185-198, doi:10.1016/S0168-1923(00)00164-7, 2000.

McCarthy, J.: A method for correcting airborne temperature data for sensor response time, J. Appl. Meteorol., 12, 211-214, doi:10.1175/1520-0450(1973)012<0211:AMFCAT>2.0.CO;2, 1973.

McLaughlin, J. P. and O'Byrne, F. D.: The role of daughter product plateout in passive radon detection, Radiat. Prot. Dosim., 7, 115119, http://rpd.oxfordjournals.org/content/7/1-4/115, 1984.

Moore, C.: Frequency response corrections for eddy correlation systems, Boundary-Layer Meteorol., 37, 17-35, doi:10.1007/BF00122754, 1986.

Nazaroff, W. W., Kong, D., and Gadgil, A. J.: Numerical investigations of the deposition of unattached ${ }^{218} \mathrm{Po}$ and ${ }^{212} \mathrm{~Pb}$ from natural convection enclosure flow, J. Aerosol Sci., 23, 339-352, doi:10.1016/0021-8502(92)90003-E, 1992.

Patil, A., Huard, D., and Fonnesbeck, C. J.: PyMC: Bayesian stochastic modelling in Python, J. Stat. Softw., 35, 1-81, doi:10.18637/jss.v035.i04, 2010.

Porstendörfer, J.: Physical parameters and dose factors of the radon and thoron decay products, Radiat. Prot. Dosim., 94, 365-373, doi:10.1093/oxfordjournals.rpd.a006512, 2001.

Powell, M. J. D.: An efficient method for finding the minimum of a function of several variables without calculating derivatives, Comput. J., 7, 155-162, doi:10.1093/comjnl/7.2.155, 1964.

Press, W. H., Teukolsky, S. A., Vetterling, W. T., and Flannery, B. P.: Numerical recipes: The art of scientific computing, Cambridge University Press, 3rd Edn., 2007.

Richardson, W. H.: Bayesian-based iterative method of image restoration, J. Opt. Soc. Am., 62, 55-59, doi:10.1364/josa.62.000055, 1972.

Rudin, L. I., Osher, S., and Fatemi, E.: Nonlinear total variation based noise removal algorithms, Physica D, 60, 259-268, doi:10.1016/0167-2789(92)90242-F, 1992.

Scheibel, H. G. and Porstendörfer, J.: Penetration measurements for tube and screen-type diffusion batteries in the ultrafine particle size range, J. Aerosol Sci., 15, 673-682, doi:10.1016/00218502(84)90005-3, 1984.

Schmithüsen, D., Chambers, S., Fischer, B., Gilge, S., Hatakka, J., Kazan, V., Neubert, R., Paatero, J., Ramonet, M., Schlosser, C., Schmid, S., Vermeulen, A., and Levin, I.: A European-wide 222Radon and 222Radon progeny comparison study, Atmos. Meas. Tech. Discuss., submitted, 2016.

Shin, W. G., Mulholland, G. W., Kim, S. C., and Pui, D. Y. H.: Experimental study of filtration efficiency of nanoparticles below $20 \mathrm{~nm}$ at elevated temperatures, J. Aerosol Sci., 39, 488-499, doi:10.1016/j.jaerosci.2008.01.006, 2008.

Slemr, F., Brunke, E.-G., Whittlestone, S., Zahorowski, W., Ebinghaus, R., Kock, H. H., and Labuschagne, C.: ${ }^{222}$ Rn-calibrated mercury fluxes from terrestrial surface of southern Africa, Atmos. Chem. Phys., 13, 6421-6428, doi:10.5194/acp-13-64212013, 2013. 
Solomon, S. B. and Ren, T.: Counting efficiencies for alpha particles emitted from wire screens, Aerosol Sci. Technol., 17, 69-83, doi:10.1080/02786829208959561, 1992.

Su, Y. F., Newton, G. J., Cheng, Y. S., and Yeh, H. C.: Experimental measurements of the diffusion coefficients and calculated sizes of Pb-212 particles, J. Aerosol Sci., 19, 767-770, doi:10.1016/0021-8502(88)90011-0, 1988.

Thomas, D., Mouret, G., Cadavid-Rodriguez, M. C., Chazelet, S., and Bémer, D.: An improved model for the penetration of charged and neutral aerosols in the 4 to $80 \mathrm{~nm}$ range through stainless steel and dielectric meshes, J. Aerosol Sci., 57, 32-44, doi:10.1016/j.jaerosci.2012.10.007, 2013.

Thomas, J. W. and Leclare, P. C.: A study of the twofilter method for radon-222, Health Phys., 18, 113-122, doi:10.1097/00004032-197002000-00002, 1970.

van der Laan, S., van der Laan-Luijkx, I. T., Zimmermann, L., Conen, F., and Leuenberger, M.: Net $\mathrm{CO}_{2}$ surface emissions at Bern, Switzerland inferred from ambient observations of $\mathrm{CO}_{2}, \delta\left(\mathrm{O}_{2} / \mathrm{N}_{2}\right)$, and ${ }^{222} \mathrm{Rn}$ using a customized radon tracer inversion, J. Geophys. Res.-Atmos., 119, 1580-1591, doi:10.1002/2013JD020307, 2014.

Vargas, A., Arnold, D., Adame, J. A., Grossi, C., HernándezCeballos, M. A., and Bolivar, J. P.: Analysis of the vertical radon structure at the Spanish "El Arenosillo" tower station, J. Environ. Radioact., 139, 1-17, doi:10.1016/j.jenvrad.2014.09.018, 2015.

Vinuesa, J.-F., Basu, S., and Galmarini, S.: The diurnal evolution of 222Rn and its progeny in the atmospheric boundary layer during the Wangara experiment, Atmos. Chem. Phys., 7, 5003-5019, doi:10.5194/acp-7-5003-2007, 2007.

Vogel, F. R., Thiruchittampalam, B., Theloke, J., Kretschmer, R., Gerbig, C., Hammer, S., and Levin, I.: Can we evaluate a finegrained emission model using high-resolution atmospheric transport modelling and regional fossil fuel $\mathrm{CO}_{2}$ observations?, Tellus B, 65, doi:10.3402/tellusb.v65i0.18681, 2013.

Wada, A., Murayama, S., Kondo, H., Matsueda, H., Sawa, Y., and Tsuboi, K.: Development of a compact and sensitive electrostatic radon-222 measuring system for use in atmospheric observation, J. Meteorol. Soc. Jpn. Ser. II, 88, 123-134, doi:10.2151/jmsj.2010-202, 2010.

Wada, A., Matsueda, H., Murayama, S., Taguchi, S., Kamada, A., Nosaka, M., Tsuboi, K., and Sawa, Y.: Evaluation of anthropogenic emissions of carbon monoxide in East Asia derived from the observations of atmospheric radon-222 over the western North Pacific, Atmos. Chem. Phys., 12, 12119-12132, doi:10.5194/acp-12-12119-2012, 2012.

Whittlestone, S. and Zahorowski, W.: Baseline radon detectors for shipboard use: development and deployment in the First Aerosol Characterization Experiment (ACE 1), J. Geophys. Res., 103, 16743-16751, doi:10.1029/98JD00687, 1998.

Whittlestone, S., Zahorowski, W., and Wasiolek, P.: High sensitivity two filter radon/thoron detectors with a wire or nylon screen as a second filter, ANSTO E Report E718, ANSTO, available at: http://apo.ansto.gov.au/dspace/bitstream/10238/376/1/ ANSTO-E-718.pdf, 1994.
Williams, A. G. and Chambers, S. D.: A history of radon measurements at Cape Grim, Baseline Atmospheric Program (Australia) 2011-2013, 16 pp., 2016.

Williams, A. G., Zahorowski, W., Chambers, S., Griffiths, A., Hacker, J. M., Element, A., and Werczynski, S.: The vertical distribution of radon in clear and cloudy daytime terrestrial boundary layers, J. Atmos. Sci., 68, 155-174, doi:10.1175/2010JAS3576.1, 2011.

Williams, A. G., Chambers, S., and Griffiths, A.: Bulk mixing and decoupling of the nocturnal stable boundary layer characterized using a ubiquitous natural tracer, Boundary-Lay. Meteorol., 149, 381-402, doi:10.1007/s10546-013-9849-3, 2013.

Winderlich, J., Chen, H., Gerbig, C., Seifert, T., Kolle, O., Lavric, J. V., Kaiser, C., Höfer, A., and Heimann, M.: Continuous lowmaintenance $\mathrm{CO}_{2} / \mathrm{CH}_{4} / \mathrm{H}_{2} \mathrm{O}$ measurements at the Zotino Tal Tower Observatory (ZOTTO) in Central Siberia, Atmos. Meas. Tech., 3, 1113-1128, doi:10.5194/amt-3-1113-2010, 2010.

Xia, Y., Sartorius, H., Schlosser, C., Stöhlker, U., Conen, F., and Zahorowski, W.: Comparison of one- and two-filter detectors for atmospheric ${ }^{222} \mathrm{Rn}$ measurements under various meteorological conditions, Atmos. Meas. Tech., 3, 723-731, doi:10.5194/amt-3723-2010, 2010.

Xia, Y., Conen, F., and Alewell, C.: Total bacterial number concentration in free tropospheric air above the Alps, Aerobiologia, 29, 153-159, doi:10.1007/s10453-012-9259-x, 2013.

Zahorowski, W. and Whittlestone, S.: A fast portable emanometer for field measurement of radon and thoron flux, Radiat. Prot. Dosim., 67, 109-120, 1996.

Zahorowski, W., Chambers, S., and Henderson-Sellers, A.: Ground based radon-222 observations and their application to atmospheric studies, J. Environ. Radioact., 76, 3-33, doi:10.1016/j.jenvrad.2004.03.033, 2004.

Zahorowski, W., Griffiths, A. D., Chambers, S. D., Williams, A. G., Law, R. M., Crawford, J., and Werczynski, S.: Constraining annual and seasonal radon-222 flux density from the Southern Ocean using radon-222 concentrations in the boundary layer at Cape Grim, Tellus B, 65, doi:10.3402/tellusb.v65i0.19622, 2013.

Zhang, H., Chen, B., Zhuo, W., and Zhao, C.: Measurements of the size distribution of unattached radon progeny by using the imaging plate, Radiat. Meas., 62, 41-44, doi:10.1016/j.radmeas.2014.01.011, 2014.

Zhang, K., Wan, H., Zhang, M., and Wang, B.: Evaluation of the atmospheric transport in a GCM using radon measurements: sensitivity to cumulus convection parameterization, Atmos. Chem Phys., 8, 2811-2832, doi:10.5194/acp-8-2811-2008, 2008.

Zhang, K., Feichter, J., Kazil, J., Wan, H., Zhuo, W., Griffiths, A. D., Sartorius, H., Zahorowski, W., Ramonet, M., Schmidt, M., Yver, C., Neubert, R. E. M., and Brunke, E.-G.: Radon activity in the lower troposphere and its impact on ionization rate: a global estimate using different radon emissions, Atmos. Chem. Phys., 11, 7817-7838, doi:10.5194/acp-11-7817-2011, 2011. 